\title{
Quantum Limits of Interferometer Topologies for Gravitational Radiation Detection
}

\author{
Haixing Miao, Huan Yang, Rana X Adhikari, and Yanbei \\ Chen \\ Division of Physics, Math, and Astronomy, California Institute of Technology, \\ Pasadena, California, 91125 \\ E-mail: rana@caltech.edu
}

\begin{abstract}
In order to expand the astrophysical reach of gravitational wave detectors, several interferometer topologies have been proposed, in the past, to evade the thermodynamic and quantum mechanical limits in future detectors. In this work, we make a systematic comparison among these topologies by considering their sensitivities and complexities. We numerically optimize their sensitivities by introducing a cost function that tries to maximize the broadband improvement over the sensitivity of current detectors. We find that frequencydependent squeezed-light injection with a hundred-meter scale filter cavity yields a good broadband sensitivity, with low complexity, and good robustness against optical loss. This study gives us a guideline for the near-term experimental research programs in enhancing the performance of future gravitational-wave detectors.
\end{abstract}

PACS numbers: 04.80.Nn, 07.05.Dz, 07.10.Fq, 07.60.Ly, 95.55.Ym

Submitted to: Class. Quantum Grav. 
Quantum Limits of Interferometer Topologies for Gravitational Radiation Detection2

\section{Introduction and Summary}

Over the past two decades, an international array of laser-interferometer gravitationalwave $(\mathrm{GW})$ detectors has been built and operated near their theoretical sensitivity limits $[1,2,3,4]$. No direct detection of gravitational waves has yet been made and this is consistent with the low event rates predicted by our knowledge of astrophysics [5].

The $2^{\text {nd }}$ generation of detectors, which are now being assembled (Advanced LIGO [6], Advanced Virgo [7], GEO-HF [8] and KAGRA [9]), are expected to improve sensitivities by a factor of $\sim 10$ compared with the first generation, and are expected to make direct detections. In order to move from the era of detections to the era of precision GW measurement, the detector sensitivities must be further improved [10].

The sensitivity of a laser interferometer gravitational-wave detector is limited by many noise sources. Among them, the quantum noise is due to ground-state fluctuations of the electromagnetic field, which beat with the laser field to produce shot noise and radiation pressure noise. For $2^{\text {nd }}$ generation detectors, quantum noise is dominant in a large fraction of the entire observation band. Furthermore, the quantum noise is at a level at which the Heisenberg Uncertainty Principle for kilogram scale masses becomes important.

Although many sources of noise can be regarded as having entered interferometer output data through "imperfections" of the interferometer, quantum noise is so tightly coupled into the gravitational wave transduction process, that improving the quantum noise often requires changing the optical configuration of the interferometer. In the past decades, several types of strategies for improving the optical configuration have been proposed within the community [11, 12]:

(i) injection of squeezed light[13, 14, 15] from the interferometer's dark port

(ii) inserting optical filters at the interferometer's input or output port

(iii) reshaping the interferometer's optical transfer function in the frequency domain

(iv) modifying the test masses' mechanical transfer function, e.g., by using the optical spring effect associated with detuned signal recycling

(v) injecting multiple carrier fields

These strategies are meant to be combined with each other in order to synthesize an optimal optical configuration. In this paper, regarding (i) above, we shall always assume that squeezed light will be injected; for (ii), depending on whether we use input or output filters, we will consider two options:

- frequency dependent squeeze angle -injecting squeezed light with an optical filter cavity $[16,17,18,19,20]$;

- frequency dependent readout - filtering the output with a cavity to measure appropriate signal quadratures at different frequencies [21, 16, 22].

Next, for each of the two options above for modifying the input-output optics, we will consider one of the following four options for the interferometer itself:

- keeping the signal-recycled configuration of Advanced LIGO [uses strategy (iv) above];

- speed-meter configurations [23, 24, 25, 26, 27], i.e., measuring a quantity that is proportional to the test mass speed at low frequencies, by inserting an additional long cavity [using strategy (iii) above]; 
- long signal recycling cavity - using a $\mathrm{km}$ scale signal recycling cavity to have a frequency-dependent response [using strategy (iii) and (iv)];

- dual-carrier scheme [28] - introducing an additional laser field to gain another readout channel; in particular, we shall also consider the so-called local-readout scheme, in which the additional field is anti-resonant in the arm cavity and resonant in the power-recycling cavity [using strategy (iii) and (iv)].

When trying to evaluate these configurations, we will include the effect of realistic optical losses and quantitatively compare these configurations against a few baseline interferometer configuration (which includes realistic levels of non-quantum noise). This differs from the proposed Einstein Telescope (ET) [29] which assumes significant reduction of the non-quantum noises at low frequencies and entirely new infrastructure with much longer arms, we are focusing on near-term upgrades to current detectors using technologies that can be deployed within the existing facilities.

We will numerically optimize the sensitivity of different configurations for this next generation detector (which we call LIGO3), with the following cost function:

$$
\mathcal{C}(\boldsymbol{x})=\left\{\int_{f_{\min }}^{f_{\max }} \mathrm{d}\left(\log _{10} f\right) \log _{10}\left[\frac{h_{\mathrm{aLIGO}}}{h_{\mathrm{LIGO} 3}(\boldsymbol{x})}\right]\right\}^{-1} .
$$

Here $\left[f_{\min }, f_{\max }\right]=[4 \mathrm{~Hz}, 4040 \mathrm{~Hz}]$ is the frequency span for the optimization; $\boldsymbol{x}$ are the set of parameters of the optical configuration that we optimize over; $h_{\text {aLIGO }}$ is the square root of the total noise power spectral density of the baseline design of Advanced LIGO (aLIGO); and $h_{\mathrm{LIGO} 3}$ is the square root of the total noise power spectral density of interferometers with various improved optical configurations. Notice that the integration variable is $\log _{10} f$ instead of $f$, which means that we want to maximize the improvement over aLIGO in the log-log scale.

The results of the numerical optimization are shown in Figures 1 and 2, where we plot the total noise spectra (the quantum noise + the classical noises) for different configurations with frequency dependent squeezing (input filtering) and frequency dependent readout (output filtering), respectively. In producing Figure 1, we assume a moderate reduction in the thermal noise and the same mass and optical power as those for aLIGO. In producing Figure 2, we assume a more optimistic reduction in the thermal noise, the mirror mass to be $150 \mathrm{~kg}$ and the maximum arm cavity power to be $3 \mathrm{MW}$. As we can see, by adding just one filter cavity to the signalrecycled interferometer (the baseline aLIGO topology), we can already obtain a substantial broadband improvement over aLIGO. Further low-frequency enhancement can be achieved by applying either the speed meter or the local-readout (dual-carrier Michelson) scheme.

The outline of this paper goes as follows: in Section 2, we summarize the basics of our quantum noise calculations; in Section 3, we introduce the interferometer topologies and the features of the configurations that we compare; in Section 4, we introduce classical noise models for $3^{\text {rd }}$ generation detectors and then compare different optical configurations by optimizing their parameters under the same cost function defined in Equation 1; in Section 6, we summarize our main results. In the appendices, we present a table of the optimized interferometer parameters, describe the non-quantum noise sources, and also define the variables used here in comparison to the previous literature on this topic. 
Quantum Limits of Interferometer Topologies for Gravitational Radiation Detection4
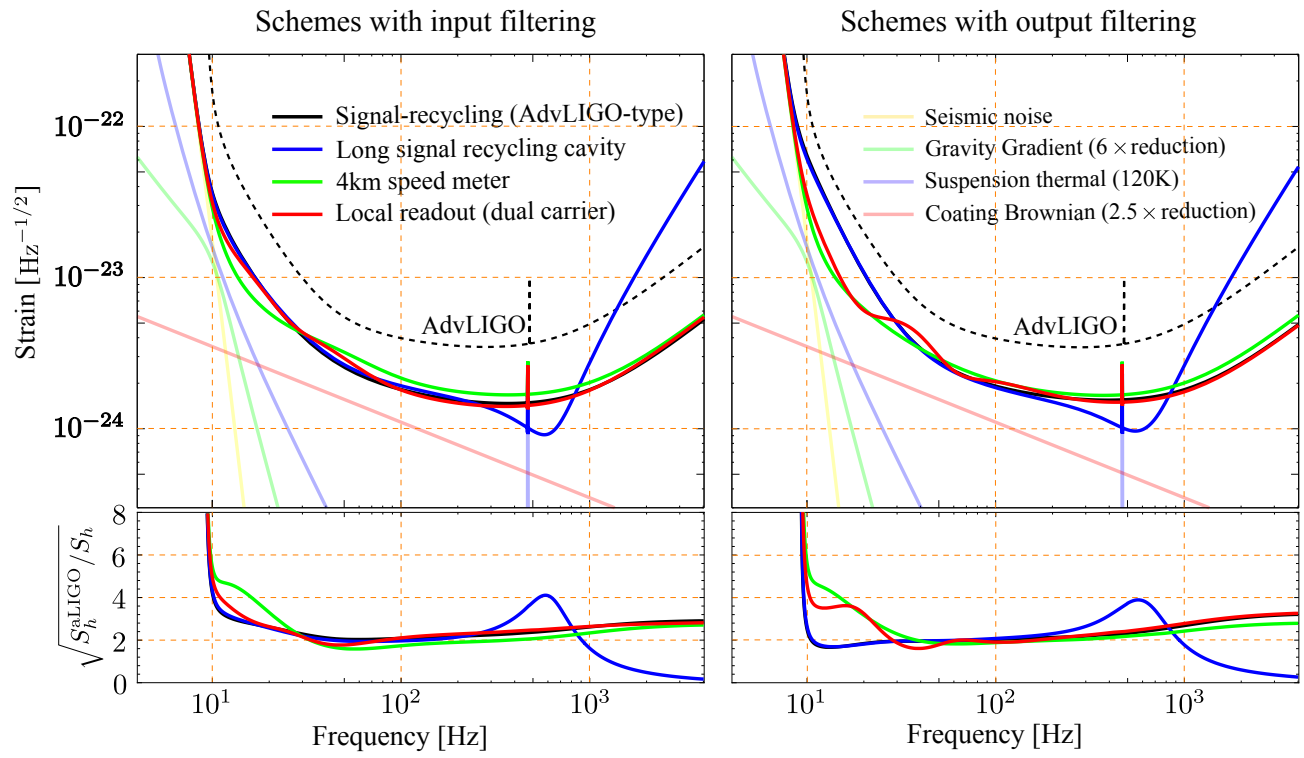

Figure 1: The optimized total noise spectrum for different schemes assuming a moderate improvement of the thermal noise compared with aLIGO baseline design. The left panel shows the case for schemes with input filtering, i.e., frequency-dependent squeezing, while the right panel shows the case for schemes with output filtering, i.e., frequency dependent readout, which is also called variational readout in the literature. The lower panels show the linear strain sensitivity improvement over Advanced LIGO.
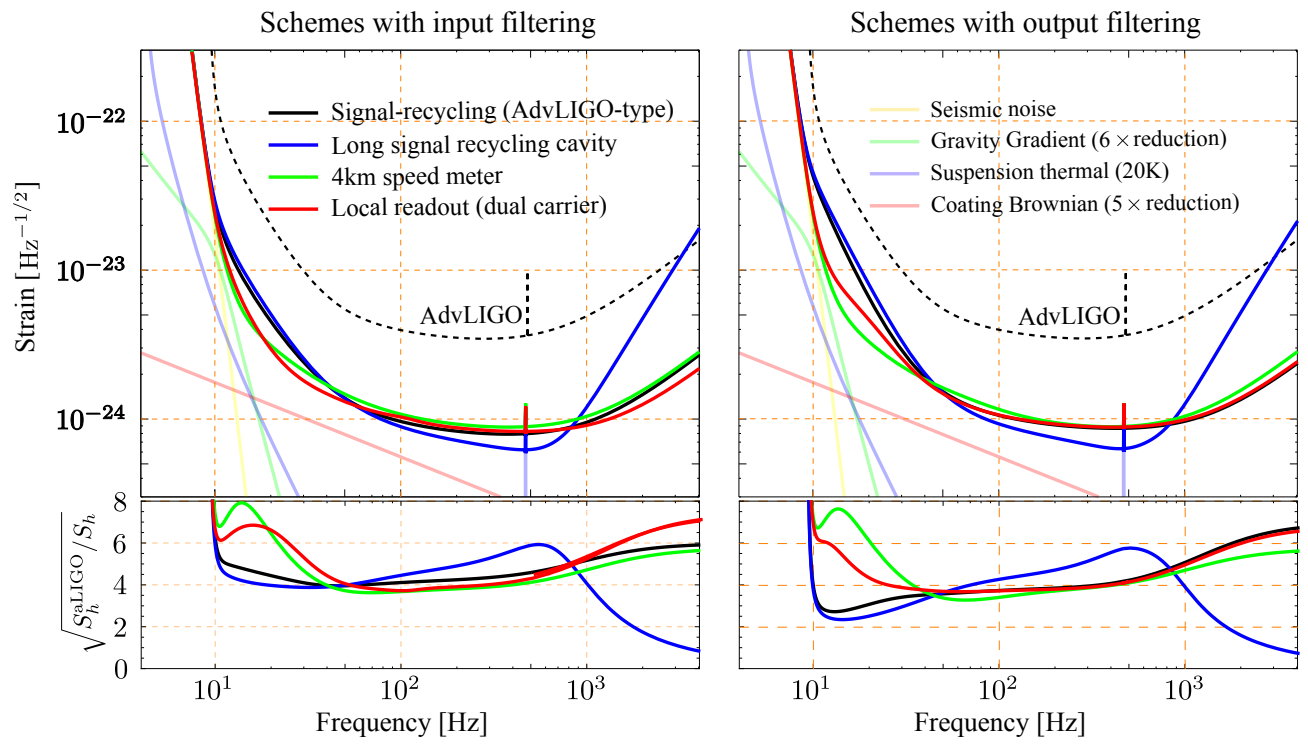

Figure 2: Optimization results for different schemes assuming more substantial thermal noise improvements, increasing the mirror mass from $40-150 \mathrm{~kg}$, and increasing the arm cavity power from $800-3000 \mathrm{~kW}$. 
Quantum Limits of Interferometer Topologies for Gravitational Radiation Detection5

\section{Basics of Quantum Noise}

In this section, we will briefly review the basics for evaluating quantum noise in a laser-interferometer gravitational-wave detector by using an input-output formalism. Additionally, we will discuss the principle behind the use of filter cavities for reducing the quantum noise. For more detail, one can refer to a recent review article [30].

\subsection{Input-Output Formalism}
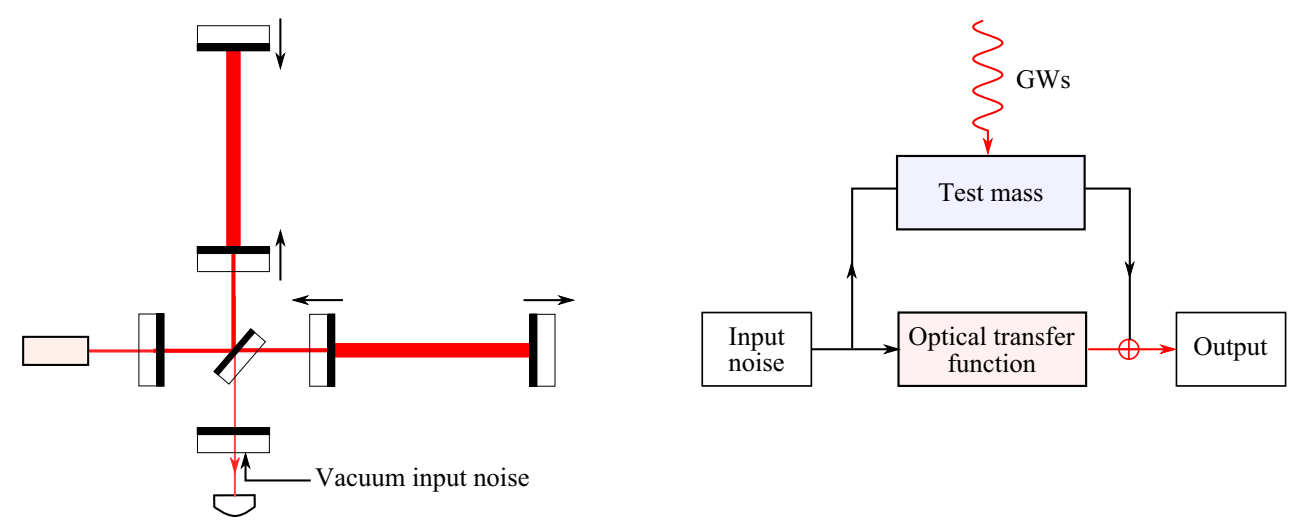

Figure 3: Schematics showing the configuration of an interferometric gravitational-wave (GW) detector (left) and the block diagram illustrating the two paths (upper and lower) through which the vacuum fluctuation propagates to the output (right).

When analyzing the quantum noise of a laser interferometer, as shown schematically in Figure 3, we assume linearity and stationarity of the system; a frequency-domain analysis can therefore be applied with the noise and signal propagating through the system via various linear transfer functions. There are two types of noise: (i) the shot noise, also called the readout noise, is the one that comes from the measurement device itself - in the context here, arising from the phase fluctuation of the light, and it usually decreases as we increase the measurement strength (the optical power). Its propagation is denoted by the lower path of the block diagram in Figure 3 (ii) the back-action noise, also called the radiation-pressure noise here, is the one that disturbs the test mass due to noise in the device, and it usually increases when the measurement strength increases. Its propagation is shown by the upper path of the diagram. In general, these two types of noise are mixed with each other. To evaluate detector sensitivity, the key is then to analyze how the noise and signal propagate and to identify those transfer functions, which gives the input-output relations.

For these interferometers, the photocurrent output $I_{\text {out }}$ that we measure is linearly proportional to a certain optical quadrature - a linear combination of the amplitude quadrature $b_{1}$ and phase quadrature $b_{2} \ddagger$ :

$$
I_{\text {out }}(\Omega) \propto b_{1}(\Omega) \sin \zeta+b_{2}(\Omega) \cos \zeta,
$$

$\ddagger$ These quadratures are related to the upper $b(\Omega)$ and lower audio sideband $b(-\Omega)$ via $b_{1}=$ $\left[b(\Omega)+b^{\dagger}(-\Omega)\right] / \sqrt{2}$ and $b_{2}=\left[b(\Omega)-b^{\dagger}(-\Omega)\right] /(i \sqrt{2})$. 
where $\zeta$ is the readout quadrature angle and can be adjusted by the phase of the local oscillator (the optical field that beats with the interferometer output). In terms of amplitude and phase quadratures, the input-output relation can generally be put into the following form:

$$
\left[\begin{array}{l}
b_{1}(\Omega) \\
b_{2}(\Omega)
\end{array}\right]=\left[\begin{array}{ll}
M_{11}(\Omega) & M_{12}(\Omega) \\
M_{21}(\Omega) & M_{22}(\Omega)
\end{array}\right]\left[\begin{array}{l}
a_{1}(\Omega) \\
a_{2}(\Omega)
\end{array}\right]+\left[\begin{array}{l}
v_{1}(\Omega) \\
v_{2}(\Omega)
\end{array}\right] h(\Omega)
$$

Here $\Omega=2 \pi f$ is the angular frequency; $b_{1}\left(a_{1}\right)$ and $b_{2}\left(a_{2}\right)$ are the output (input) amplitude quadrature and phase quadrature, respectively; $M_{i j}$ are the elements of the transfer matrix, which depend on the specific optical configuration; $v_{i}$ quantify the detector response to the gravitational-wave strain $h$. More compactly, one can rewrite the input-output relation in a vector form: $\boldsymbol{b}(\Omega)=\mathbf{M}(\Omega) \boldsymbol{a}(\Omega)+\boldsymbol{v}(\Omega) h(\Omega)$. Different configurations will have different transfer matrices and response functions to the gravitational-wave signal - thus different input-output relations. In the following sections, we will see an interesting assortment of them.

Given the above input-output relation and the readout angle $\zeta$ of the homodyne detection by adjusting the local oscillator phase, the output current of the photodiode is proportional to $y(\Omega)=\boldsymbol{d}_{\zeta}^{\mathrm{T}} \boldsymbol{b}(\Omega)$, namely,

$$
y(\Omega)=\boldsymbol{d}_{\zeta}^{\mathrm{T}} \mathbf{M}(\Omega) \boldsymbol{a}(\Omega)+\boldsymbol{d}_{\zeta}^{\mathrm{T}} \boldsymbol{v}(\Omega) h(\Omega),
$$

where the readout vector is defined as $\boldsymbol{d}_{\zeta} \equiv(\sin \zeta, \cos \zeta)^{\mathrm{T}}$. The first term is the quantum noise, while the second term is the output response to the GW signal. The detector sensitivity is quantified by the noise power spectral density $\S$ (normalized with respect to the GW strain $h$ ):

$$
S_{h}(\Omega)=\frac{\boldsymbol{d}_{\zeta}^{\mathrm{T}} \mathbf{M}(\Omega) \mathbf{S}(\Omega) \mathbf{M}^{\dagger}(\Omega) \boldsymbol{d}_{\zeta}}{\left|\boldsymbol{d}_{\zeta}^{\mathrm{T}} \boldsymbol{v}(\Omega)\right|^{2}},
$$

where $\mathbf{S}$ is the noise spectral-density matrix for the input amplitude quadrature $a_{1}$ and the phase quadrature $a_{2}-\left\langle a_{i}(\Omega) a_{j}^{\dagger}\left(\Omega^{\prime}\right)\right\rangle_{\text {sym }} \equiv \pi S_{i j}(\Omega) \delta\left(\Omega-\Omega^{\prime}\right)(i, j=1,2)$, and in particular for non-squeezed light (vacuum) input, its elements are $S_{11}=S_{22}=1$ and $S_{12}=S_{21}=0$ (uncorrelated amplitude and phase noise).

Taking a broadband, resonant sideband extraction (RSE), tuned dual-recycled interferometer (the baseline configuration of aLIGO) for example, the input-output relation is given by [16]:

$$
\left[\begin{array}{l}
b_{1} \\
b_{2}
\end{array}\right]=e^{2 i \phi}\left[\begin{array}{cc}
1 & 0 \\
-\mathcal{K}(\Omega) & 1
\end{array}\right]\left[\begin{array}{l}
a_{1} \\
a_{2}
\end{array}\right]+e^{i \phi}\left[\begin{array}{c}
0 \\
\sqrt{2 \mathcal{K}(\Omega)}
\end{array}\right] \frac{h(\Omega)}{h_{\mathrm{SQL}}} .
$$

Here we have introduced:

$$
\phi \equiv \arctan (\Omega / \gamma), \quad \mathcal{K}(\Omega) \equiv \frac{2 \gamma \iota_{c}}{\Omega^{2}\left(\Omega^{2}+\gamma^{2}\right)}, \quad h_{\mathrm{SQL}} \equiv \sqrt{\frac{8 \hbar}{m \Omega^{2} L^{2}}}
$$

with $\gamma$ the arm cavity bandwidth, $L$ the arm cavity length, $\iota_{c} \equiv 8 \omega_{0} P_{c} /(m L c)$ for quantifying the measurement strength, $\omega_{0}$ the laser angular frequency, and $P_{c}$ the arm cavity power. If we measure the phase quadrature by choosing the readout phase to be $\zeta=0$, the corresponding noise power spectral density will be:

$$
S_{h}(\Omega)=\left[\mathcal{K}(\Omega)+\frac{1}{\mathcal{K}(\Omega)}\right] \frac{h_{\mathrm{SQL}}^{2}}{2} \geq h_{\mathrm{SQL}}^{2}=\frac{8 \hbar}{m \Omega^{2} L^{2}} .
$$

$\S$ The single-sided power spectral density $S_{A}(\Omega)$ for any quantity $A$ is defined by [cf. Equation (22) in [16]]: $\left\langle A(\Omega) A^{\dagger}\left(\Omega^{\prime}\right)\right\rangle_{\mathrm{sym}}=\frac{1}{2}\left\langle A(\Omega) A^{\dagger}\left(\Omega^{\prime}\right)+A^{\dagger}\left(\Omega^{\prime}\right) A(\Omega)\right\rangle=\frac{1}{2} 2 \pi S_{A}(\Omega) \delta\left(\Omega-\Omega^{\prime}\right)$. 
Quantum Limits of Interferometer Topologies for Gravitational Radiation Detection7

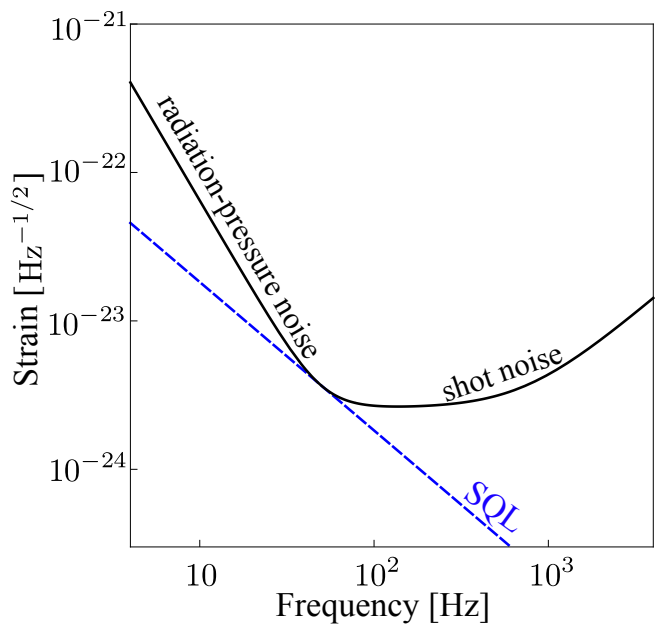

Figure 4: The quantum-noise spectral density $S_{h}^{1 / 2}$ for a broadband RSE interferometer given the same specification of aLIGO $-m=40 \mathrm{~kg}$ and $P_{c}=800 \mathrm{~kW}$.

The first term, proportional to the optical power $\left(\mathcal{K} \propto P_{c}\right)$, is the radiationpressure noise and comes from the fluctuation of the input amplitude quadrature $a_{1}$; the second term, inversely proportional to the optical power, is the shot noise and comes from the fluctuation of the input phase quadrature $a_{2}$. In this simple scenario, the sensitivity is limited by the standard quantum limit (SQL) - the benchmark for the strength of quantum noise [31]. In Figure 4 , we plot $S_{h}^{1 / 2}(\Omega)$ - the radiation-pressure noise dominates at low frequencies and the shot noise dominates at high frequencies. 
Quantum Limits of Interferometer Topologies for Gravitational Radiation Detection8

\section{Optical Topologies}

In this section, we briefly describe the strategies for configuration improvements that will be used in Section 4. We will compute the corresponding quantum noise spectrum using the input-output formalism introduced in Reference 2.

\subsection{Frequency-dependent squeezing angle (input filtering)}

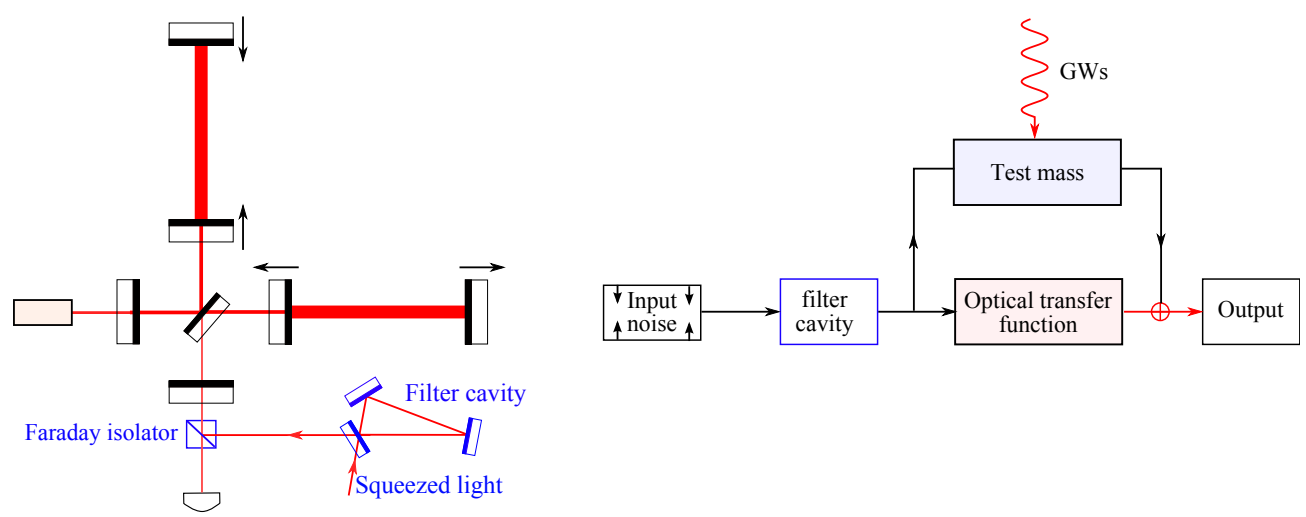

Figure 5: Schematic showing the frequency dependent squeezed light phase angle rotation scheme (left) and its associated block diagram (right).

Unlike the vacuum state, for which the cross spectral density matrix for any two orthogonal field quadratures is the identity matrix, squeezed light has the following cross spectral density matrix for the quadratures $b_{1}$ and $b_{2}$ :

$$
\begin{aligned}
& {\left[\begin{array}{ll}
S_{11} & S_{12} \\
S_{21} & S_{22}
\end{array}\right] } \\
= & {\left[\begin{array}{cc}
\cosh 2 r-\sinh 2 r \cos 2 \varphi & -\sinh 2 r \sin 2 \varphi \\
-\sinh 2 r \sin 2 \varphi & \cosh 2 r+\sinh 2 r \cos 2 \varphi
\end{array}\right] }
\end{aligned}
$$

where $r$ is called the squeezing factor $\left(10 \mathrm{~dB}\right.$ squeezing means that $\left.e^{2 r}=10\right)$ and $\varphi$ is the squeezing angle. If we define the $\zeta$ quadrature [see Section 2 for details] as

$$
b_{\zeta}=b_{1} \cos \zeta+b_{2} \sin \zeta,
$$

and if we denote by $S_{\zeta}$ the spectral density of $b_{\zeta}$, then the matrix becomes

$$
S_{\zeta}=S_{11} \cos ^{2} \zeta+2 S_{12} \cos \zeta \sin \zeta+S_{22} \sin ^{2} \zeta \text {. }
$$

In particular, $S_{\varphi}=e^{-2 r}$, which means the $\varphi$-quadrature (often referred to as the squeezed quadrature) has a fluctuation that is $e^{-r}$ the level of vacuum (in amplitude), while the $\pi / 2+\varphi$ quadrature orthogonal to $\varphi$-quadrature (often referred to as the antisqueezed quadrature) has $S_{\varphi}=e^{+2 r}$, which is $e^{+r}$ the level of vacuum fluctuation.

The above picture exists for each audio sideband frequency $\Omega$. Frequencydependent squeezed light describes a state that has different squeeze factors and/or angles for each frequency. As schematically shown in Figure 5, such a frequency dependence can be realized, for example, by injecting frequency-independent squeezed light into a Fabry-Perot cavity with a linewidth and detuning frequency comparable to the audio frequencies of interest. If we define $b_{1,2}$ as the quadrature fields of the 
output, and $a_{1,2}$ the quadratures of the input field, the cavity has an input-output relation of

$$
\left[\begin{array}{l}
b_{1} \\
b_{2}
\end{array}\right]=e^{\frac{i\left(\alpha_{+}-\alpha_{-}\right)}{2}}\left[\begin{array}{cc}
\cos \frac{\alpha_{+}+\alpha_{-}}{2} & -\sin \frac{\alpha_{+}+\alpha_{-}}{2} \\
\sin \frac{\alpha_{+}+\alpha_{-}}{2} & \cos \frac{\alpha_{+}+\alpha_{-}}{2}
\end{array}\right]\left[\begin{array}{l}
a_{1} \\
a_{2}
\end{array}\right],
$$

with $\alpha_{ \pm}$is defined as

$$
e^{i \alpha_{ \pm}} \equiv \frac{i \gamma \mp \Omega-\Delta}{i \gamma \pm \Omega-\Delta},
$$

where $\Delta$ and $\gamma$ are the detuning frequency and bandwidth of the filter cavity, respectively. As indicated by Equation 12, the quadratures undergo a frequency dependent rotation of $\left(\alpha_{+}+\alpha_{-}\right) / 2$. This converts a frequency independent squeezed vacuum into one with a frequency dependent squeezing angle.

With the correct frequency dependence, one can rotate the squeezing angle such that the quantum noise is reduced by a factor $e^{2 r}$ over the entire frequency band, namely (in the case of the broadband RSE interferometer)

$$
S_{h}^{\mathrm{opt}}(\Omega)=e^{-2 r}\left[\mathcal{K}(\Omega)+\frac{1}{\mathcal{K}(\Omega)}\right] \frac{h_{\mathrm{SQL}}^{2}}{2} .
$$

This is the optimum performance that can be realized with frequency dependent squeezed light injection.

Figure 6 shows the resulting noise spectrum in the lossless case. As we can see, the squeezing angle rotates in such a way that at low frequencies the fluctuation in the amplitude quadrature is squeezed - thus reducing the radiation-pressure noise, while at high frequencies the phase quadrature is squeezed - thus reducing the shot noise. In order to achieve the desired rotation of squeezing angle, the filter cavity needs to have a frequency bandwidth that is near the frequency where the radiation pressure noise is comparable to the shot noise.

The frequency dependence of a series of such filter cavities as well as the concomitant parameters required for realizing this frequency dependence has been derived in [25]. In practice, however, the complexity of using the "optimal" number of cavities and the performance degradation which comes from optical losses, leads one to use a sub-optimal number of cavities; the resulting degradation of the astrophysical sensitivity is negligible.

3.1.1. The Impact of Optical Scatter Loss So far, we have been considering the ideal case without optical loss. Here we provide a qualitative understanding of how loss in the filter cavity affects the sensitivity of input filtering. Basically, the optical loss introduces additional (vacuum) noise that is uncorrelated with the input squeezed light:

$$
\begin{aligned}
& a_{1}^{\prime}=\sqrt{\mathcal{E}} n_{1}+\sqrt{1-\mathcal{E}} a_{1}, \\
& a_{2}^{\prime}=\sqrt{\mathcal{E}} n_{2}+\sqrt{1-\mathcal{E}} a_{2},
\end{aligned}
$$

where $\mathcal{E}$ quantifies the total optical loss of the filter cavity and $n_{1,2}$ are the associated noise terms in the amplitude and phase quadratures. These noise sources will degrade the squeezing. For example, the amplitude squeezed light originally has $S_{11}=e^{-2 r}$ with $r>0$. Introducing optical loss according to Equation 15, it becomes:

$$
S_{11}^{\prime}=(1-\mathcal{E}) e^{-2 r}+\mathcal{E} .
$$



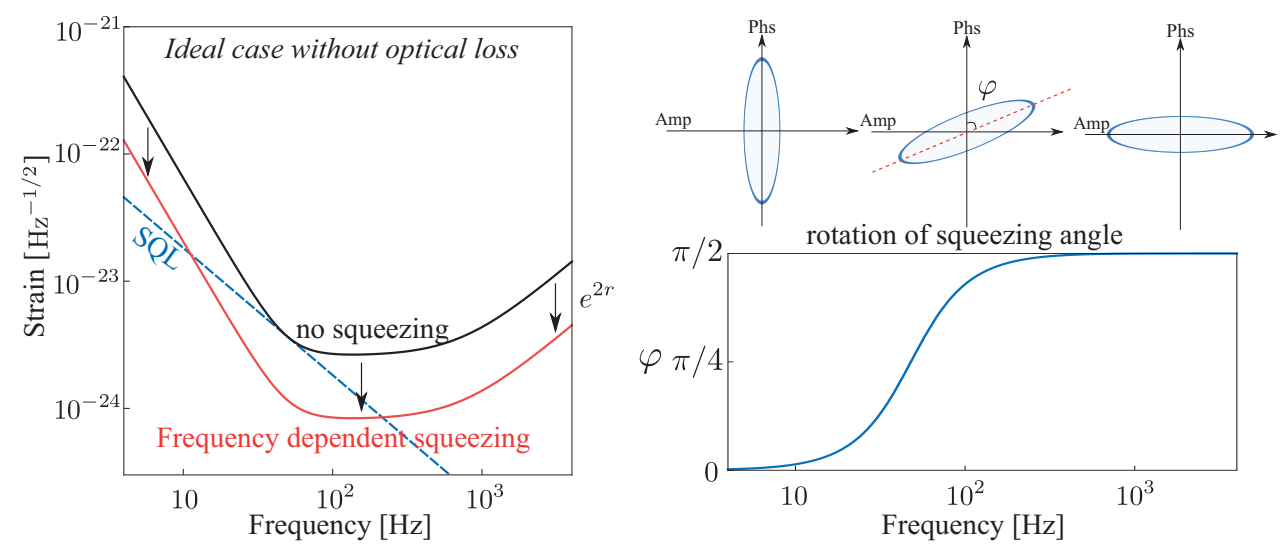

Figure 6: Noise spectrum for frequency dependent squeezing (left) and illustration of the rotation of the squeezing angle (right).

For a completely lossy case with $\mathcal{E}=1$, we have $S_{11}=1$ and the squeezing simply vanishes.

The squeezed light at different audio frequencies experiences different levels of optical loss from the filter cavity. The low frequency part enters the cavity and circulates multiple times, while the high frequency part barely enters the cavity. Therefore, the optical loss affects the low frequency part most significantly (refer to Appendix $\mathrm{C}$ for a detailed discussion). In terms of the noise power spectrum, we approximately have:

$$
S_{h}(\Omega)=\left\{\left[(1-\mathcal{E}) e^{-2 r}+\mathcal{E}\right] \mathcal{K}(\Omega)+\frac{e^{-2 r}}{\mathcal{K}(\Omega)}\right\} \frac{h_{\mathrm{SQL}}^{2}}{2},
$$

in contrast to Equation 14. Compared with the ideal frequency dependent squeezing case, the low frequency radiation pressure noise increases due to the optical loss and the high frequency shot noise remains almost the same. In Figure 7, we show the effect of optical loss. In producing the figure, we have assumed a total optical loss of $20 \%$, which is equivalent to a round trip loss of $40 \mathrm{ppm}$ given a filter cavity input mirror transmittance of $T_{f}=200 \mathrm{ppm}$.

3.1.2. The effect of parameter variations of the filter cavity Apart from the optical loss, there are other imperfections of the filter cavity that will degrade the sensitivity. In particular, here we consider the effect of variations in the parameters of the filter cavity, which make the bandwidth $\gamma_{f}$, or equivalently the input mirror transmittance $T_{f}$, and the detuning $\Delta_{f}$ deviate from their optimal value, i.e., $T_{f}=T_{f}^{\text {opt }}+\delta T_{f}$ and $\Delta_{f}=\Delta_{f}^{\mathrm{opt}}+\delta \Delta_{f}$.

As shown in Appendix D, such a parameter variation will mainly decrease the lowfrequency sensitivity (for a reason similar to the effect of loss), and we approximately have:

$$
S_{h}(\Omega) \approx S_{h}^{\mathrm{opt}}(\Omega)+\sinh 2 r\left[\left(\frac{\delta T_{f}}{T_{f}^{\mathrm{opt}}}\right)^{2}+\left(\frac{\delta \Delta_{f}}{\Delta_{f}^{\mathrm{opt}}}\right)^{2}\right] \mathcal{K}(\Omega) h_{\mathrm{SQL}}^{2}
$$



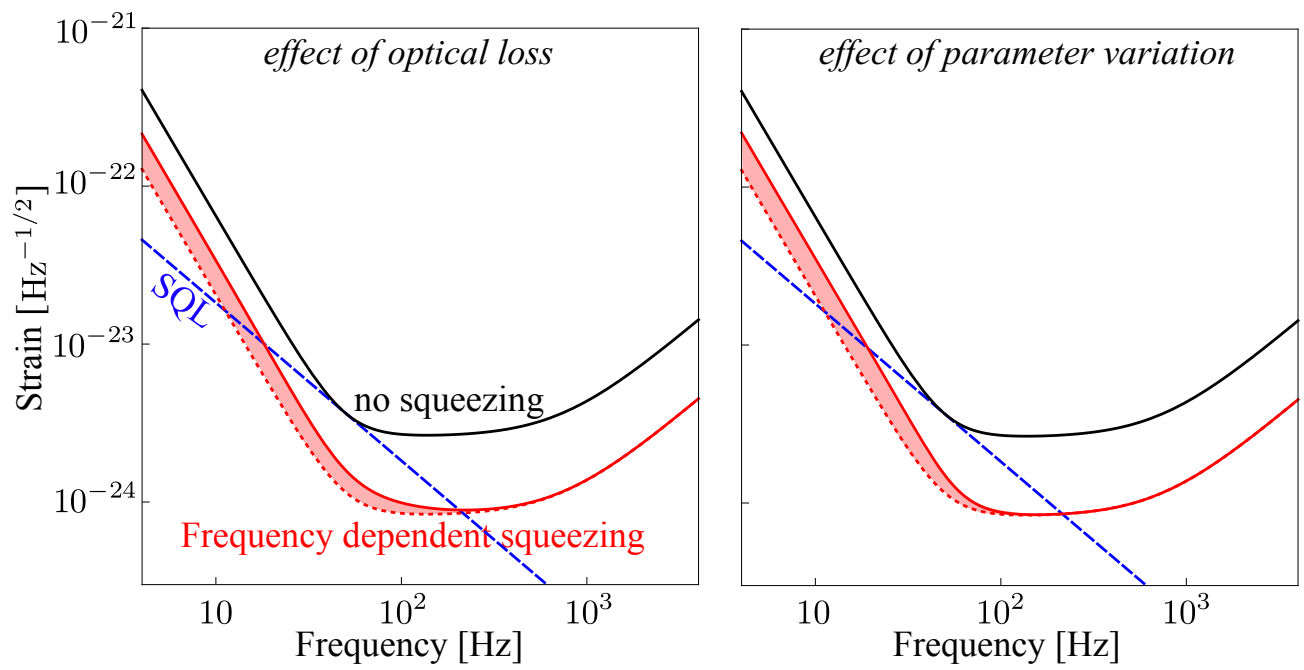

Figure 7: The effect of optical loss in the filter cavity (left) and the effect of parameter variation of the filter cavity (right) in the case of input filtering. The shaded regions illustrate the degradation of sensitivity as losses are added. Here we have assumed the total optical loss $\mathcal{E}=20 \%$ and parameter variations of $\delta T_{f} / T_{f}^{\mathrm{opt}}=\delta \Delta_{f} / \Delta_{f}^{\mathrm{opt}}=10 \%$.

If the relative error in the transmittance and the detuning can be as low as $100 \mathrm{ppm}$, namely $\delta T_{f} / T_{f}^{\mathrm{opt}} \sim 10^{-4}, \delta \Delta_{f} / \Delta_{f}^{\mathrm{opt}} \sim 10^{-4}$, we have $S_{h} \approx S_{h}^{\mathrm{opt}}+10^{-7} \mathcal{K} h_{\mathrm{SQL}}^{2}$ for $10 \mathrm{~dB}$ squeezing, which is a negligible deviation from the optimal one. In Figure 7 , we illustrate this effect with an exaggerated variation of $10 \%$.

\subsection{Frequency dependent readout phase (output filtering)}

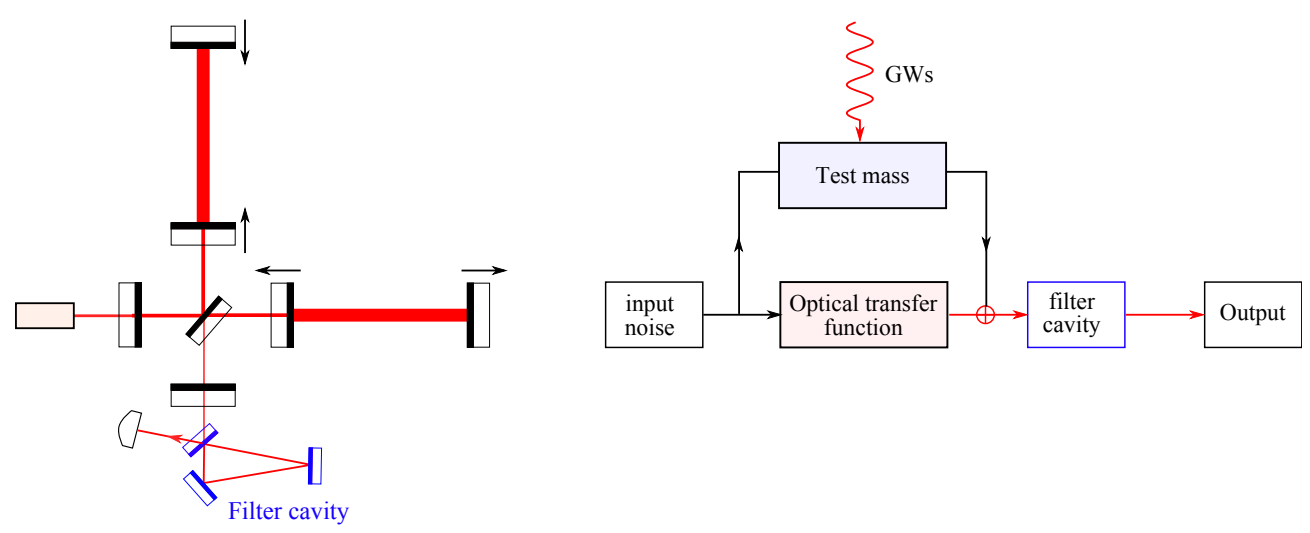

Figure 8: Schematic optical layout of the frequency dependent (or variational) readout scheme (left) and its associated block diagram (right).

A closely related counterpart to the frequency dependent squeezed light injection is the frequency dependent readout angle and, as shown schematically in Figure 8, it uses an optical cavity to filter the detector output allowing one to measure different optical quadratures at different frequencies. The filter cavity has the same 
functionality as in the case of the frequency dependent squeezing with the difference that it filters the outgoing fields at the interferometer output instead of the noise fields (or squeezed light) entering from the dark port. This scheme can be used to coherently cancel the radiation-pressure noise at low frequencies [16]. To illustrate how this works, we use the interferometer for which the input-output relation is given by Equation 6:

$$
\begin{aligned}
b_{\zeta}(\Omega)= & b_{1}(\Omega) \sin \zeta+b_{2}(\Omega) \cos \zeta \\
= & e^{2 i \phi}[\sin \zeta-\mathcal{K}(\Omega) \cos \zeta] a_{1}(\Omega)+e^{2 i \phi} \cos \zeta a_{2}(\Omega) \\
& +e^{i \phi} \cos \zeta \sqrt{2 \mathcal{K}(\Omega)} \frac{h(\Omega)}{h_{\mathrm{SQL}}}
\end{aligned}
$$

Here the first term, proportional to $a_{1}$, is the radiation pressure noise; the second term, proportional to $a_{2}$, is the shot noise; the third term is the signal. As we can see, if the quadrature angle $\zeta$ has the following frequency dependence:

$$
\tan \zeta=\mathcal{K}(\Omega),
$$

the radiation pressure term would be canceled, and give rise to a shot noise only noise floor. Since the phase for the local oscillator is usually fixed, before beating with the local oscillator we need to rotate the output quadratures with a filter cavity to achieve such a frequency-dependent quadrature readout.

The resulting noise spectrum for this scheme is simply:

$$
S_{h}(\Omega)=\frac{1}{\mathcal{K}(\Omega)} \frac{h_{\mathrm{SQL}}^{2}}{2} .
$$

If we simultaneously inject phase squeezed light, we will have:

$$
S_{h}^{\mathrm{opt}}(\Omega)=\frac{e^{-2 r}}{\mathcal{K}(\Omega)} \frac{h_{\mathrm{SQL}}^{2}}{2} .
$$

In Figure 9, we plot the noise spectrum in the ideal lossless case with the low frequency radiation pressure noise completely evaded. In reality, due to optical losses, such a cancellation cannot be perfect. In the numerical optimization, we will take into account the optical loss and optimize the parameters for the filter cavity.

3.2.1. The Effects of Optical Scatter Loss For the frequency dependent readout scheme, the additional noise introduced by optical loss influences the output and modifies the input-output relation in the following way:

$$
\begin{aligned}
{\left[\begin{array}{l}
b_{1}^{\prime} \\
b_{2}^{\prime}
\end{array}\right]=\sqrt{\mathcal{E}}\left[\begin{array}{l}
n_{1} \\
n_{2}
\end{array}\right] } & +\sqrt{1-\mathcal{E}} e^{2 i \phi}\left[\begin{array}{cc}
1 & 0 \\
-\mathcal{K} & 1
\end{array}\right]\left[\begin{array}{l}
a_{1} \\
a_{2}
\end{array}\right] \\
& +\sqrt{1-\mathcal{E}} e^{i \phi}\left[\begin{array}{c}
0 \\
\sqrt{2 \mathcal{K}}
\end{array}\right] \frac{h}{h_{\mathrm{SQL}}} .
\end{aligned}
$$

Due to the presence of uncorrelated noise, the condition in Equation 21 no longer provides radiation-pressure noise cancellation. By optimizing the quadrature angle $\zeta$ for the tuned interferometer with phase squeezed light injection, one can find that the optimal sensitivity, in contrast to Equation 23, reads:

$$
S_{h}(\Omega)=\left[\frac{\eta e^{2 r} \mathcal{K}(\Omega)}{\eta+e^{2 r}}+\frac{e^{-2 r}+\eta}{\mathcal{K}(\Omega)}\right] \frac{h_{\mathrm{SQL}}^{2}}{2}
$$

with $\eta \equiv \mathcal{E} /(1-\mathcal{E}) \approx \mathcal{E}$. The effect of loss is illustrated in Figure 10. As we can see, the low-frequency performance is very fragile, and we end up with a sensitivity similar to the input filtering case, given the same level of loss. 
Quantum Limits of Interferometer Topologies for Gravitational Radiation Detection13

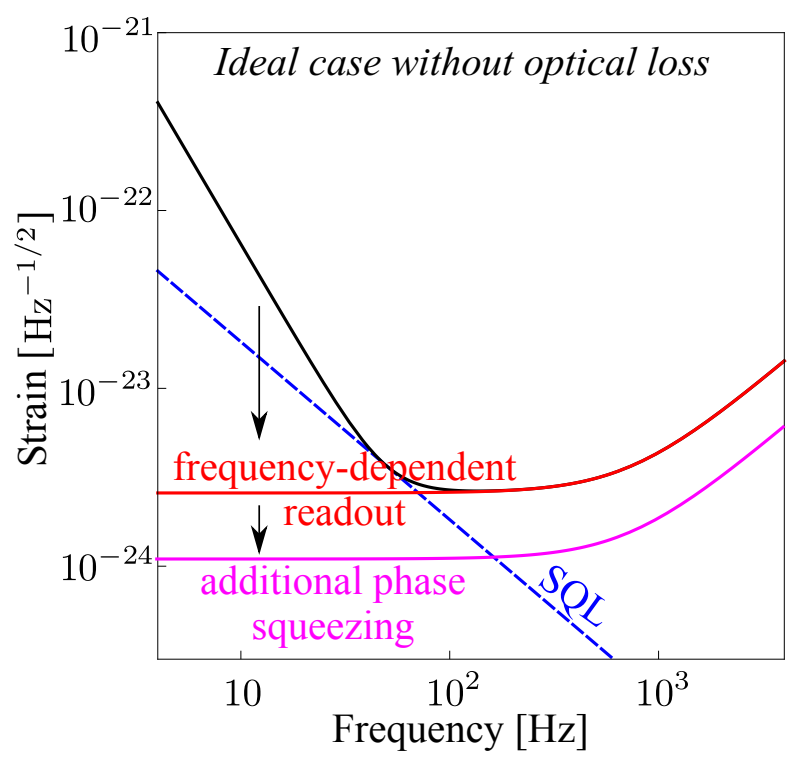

Figure 9: Noise spectra for the frequency dependent readout scheme without (red curve) and with (purple curve) additional phase squeezed light injection.
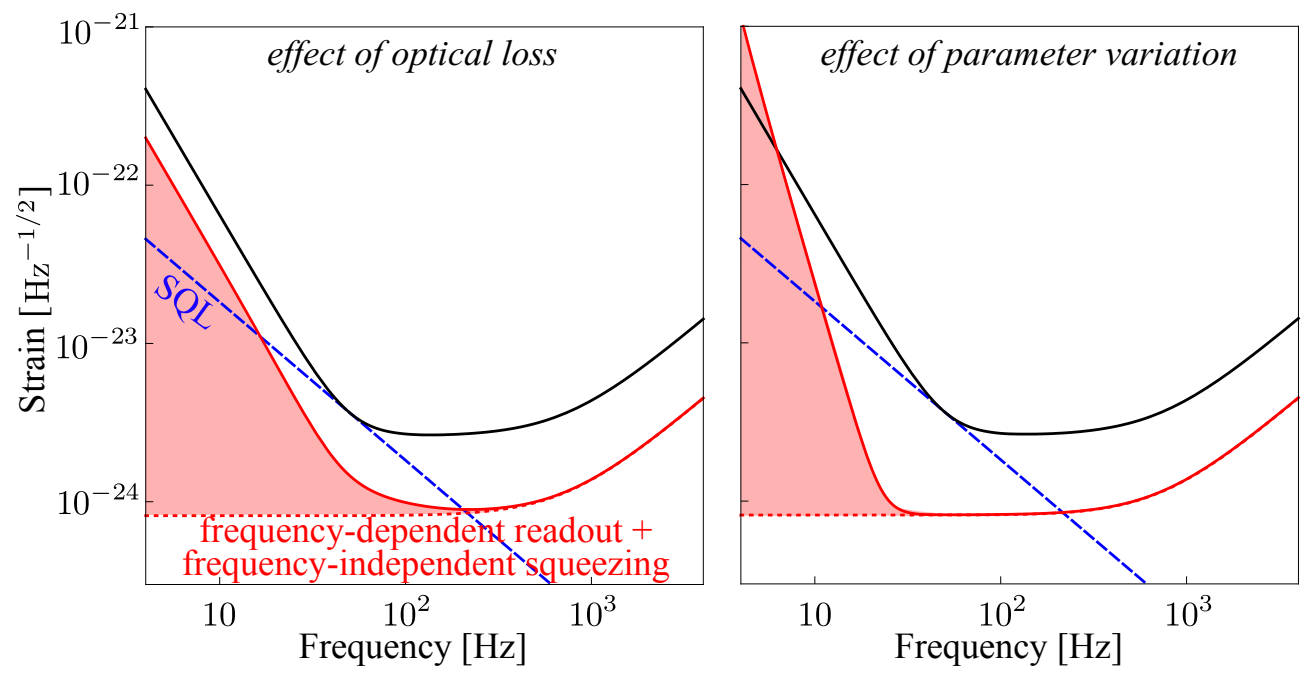

Figure 10: The effect of optical loss in the filter cavity (left) and the effect of parameter variation of the filter cavity (right) in the case of frequency-dependent readout (output filtering). Similar to the case shown in Figure 7, the shaded areas denote the degradation of sensitivity. We have used a total optical loss of $\mathcal{E}=20 \%$. In contrast, the parameter variation is chosen to be only $\delta T_{f} / T_{f}^{\mathrm{opt}}=\delta \Delta_{f} / \Delta_{f}^{\mathrm{opt}}=10^{-4}$ in order to produce reasonable sensitivity, as it is much more sensitive than input filtering. 
3.2.2. The effect of parameter variation in the filter cavity As shown in Appendix $\mathrm{D}$, the parameter variation of the filter cavity results in the following sensitivity

$$
S_{h} \approx S_{h}^{\mathrm{opt}}+\left(2 e^{4 r} \mathcal{K}+e^{8 r} \mathcal{K}^{3}\right)\left[\left(\frac{\delta T_{f}}{T_{f}^{\mathrm{opt}}}\right)^{2}+\left(\frac{\delta \Delta_{f}}{\Delta_{f}^{\mathrm{opt}}}\right)^{2}\right] h_{\mathrm{SQL}}^{2} .
$$

Since $\mathcal{K} \gg 1$, by comparing Equation 19 with Equation 26, we can see that the output filtering is more susceptible to parameter variation than input filtering, which is illustrated in Figure 10.

\subsection{Long Signal-Recycling Cavity}
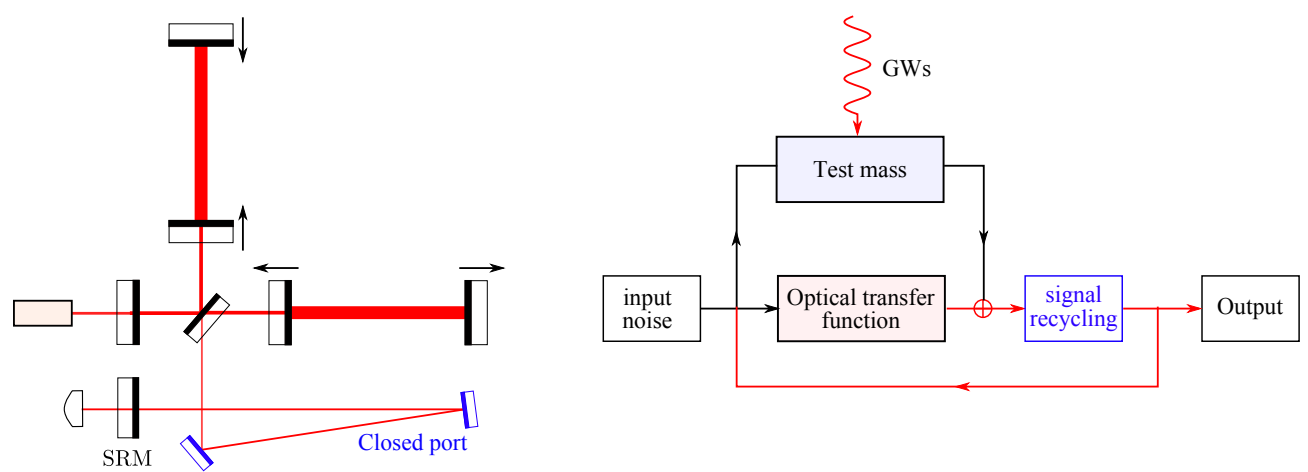

Figure 11: Schematic optical layout showing the long signal recycling cavity scheme (left) and its associated block diagram (right). The signal recycling mirror coherently reflects back the signal, forming a feedback loop as indicated in the block diagram.

In this subsection, we discuss the idea of long signal-recycling cavity, and one can refer to Ref. [32] and references therein for an overview of different recycling techniques applied in the context of GW detectors. In the usual case when the beam splitter and the signal recycling mirror are close to each other, the signal recycling cavity is relatively short (order of 10 meters) and one can ignore the phase accumulated in this cavity by the audio sidebands: $\Omega L_{\mathrm{sr}} / c \approx 0$ with $L_{\mathrm{sr}}$ being the length of the signalrecycling cavity. We can therefore treat the signal-recycling cavity as an effective compound mirror with complex transmissivity and reflectivity, which is the approach applied in [33]. With a long signal recycling cavity, however, $\Omega L_{\mathrm{sr}} / c$ is not negligible and different sidebands pick up different phase shifts. Specifically, the transfer function matrix for the quadratures due to the free propagation in the signal recycling cavity is given by:

$$
e^{i \Omega \tau_{\mathrm{sr}}}\left[\begin{array}{cc}
\cos \Delta \tau_{\mathrm{sr}} & -\sin \Delta \tau_{\mathrm{sr}} \\
\sin \Delta \tau_{\mathrm{sr}} & \cos \Delta \tau_{\mathrm{sr}}
\end{array}\right]
$$

with $\tau_{\mathrm{sr}} \equiv L_{\mathrm{sr}} / c$ and $\Delta$ being the detuning frequency of the signal recycling cavity. One can then apply the standard procedure to derive the input-output relation for this scheme. In general, the final expression is quite lengthy and not illuminating, so we will not show it here, and will evaluate its noise spectrum numerically. There is one interesting special case which allows an intuitive understanding. It is when the signal-recycling detuning phase is equal to $\pi / 2$. In this case, the coupled cavity, 
formed by the signal-recycling cavity and the arm cavity, has two resonant frequencies located symmetrically around the carrier frequency with their frequency separation determined by the ITM transmissivity. This case has two advantages: firstly, it allows an equal and balanced enhancement of both the upper and lower sidebands, in contrast to the conventional signal-recycling interferometer; secondly, there is no optical-spring effect, as the signal-recycling cavity is tuned, and the test-mass dynamics is therefore not modified. Such a case is exactly equivalent to the twin signal-recycling scheme theoretically studied by Thüring et al. [34] and experimentally demonstrated by Gräf et al. [35], which are motivated by the above mentioned two advantages.

\subsection{Speed Meter with Sloshing Cavity}

The motivation for the speed meter originates from the perspective of viewing the gravitational-wave detector as a quantum measurement device. Normally, we measure the test mass position at different times to infer the gravitational-wave signal. However, position is not a conserved dynamical quantity of a free mass. According to quantum measurement theory [31], such a measurement process will inevitably introduce additional back action on to the test mass. In the context here, the back action is the radiation-pressure noise. In order to evade the back action, one needs to measure the conserved dynamical quantities of the test mass: the momentum or the energy. Since the momentum is proportional to the speed, the speed meter can therefore detect gravitational wave without being limited by the radiation-pressure noise [23].
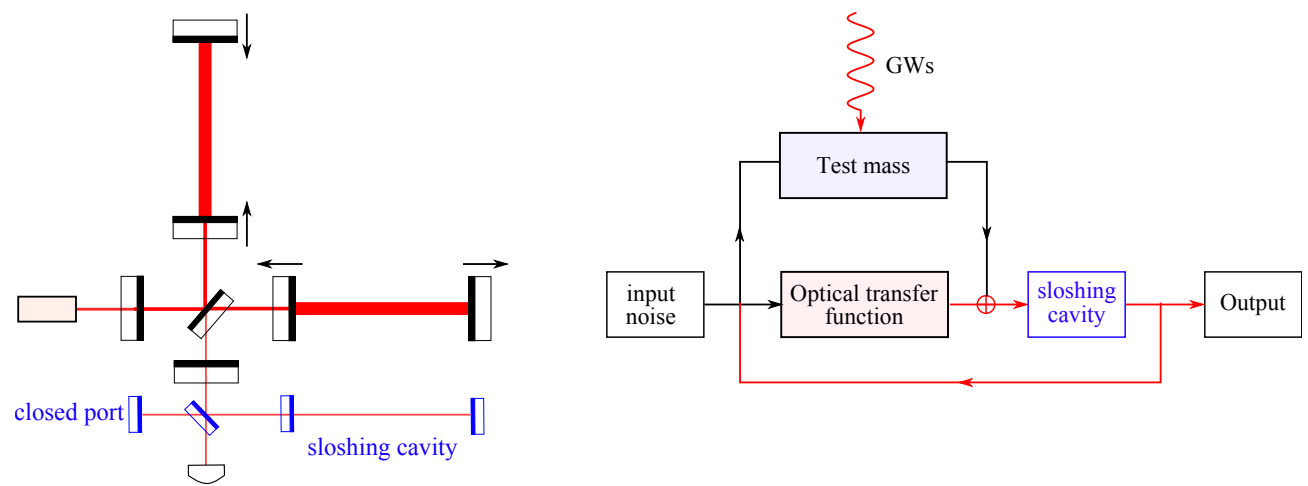

Figure 12: Schematics showing the speed-meter configuration (left) and its block diagram (right).

There are several speed-meter configurations, e.g., the Sagnac interferometer [36, $37,26,27]$ and a recent proposed scheme by using different polarizations [38]. In Figure 12, we show one particular variant, which is proposed in [25], by using a sloshing cavity. We can gain a qualitative understanding of how such a scheme allows us to measure the speed of the test mass. Basically, the information of test mass position at an early moment is stored in the sloshing cavity, and it is coherently superimposed (but with a minus sign due to the phase shift in the tuned cavity) with the output of the interferometer which contains the current test mass position. The sloshing happens at a frequency that is comparable to the detection frequency, and the superposed output is, therefore, equal to the derivative of the test-mass position, i. e., the speed. 


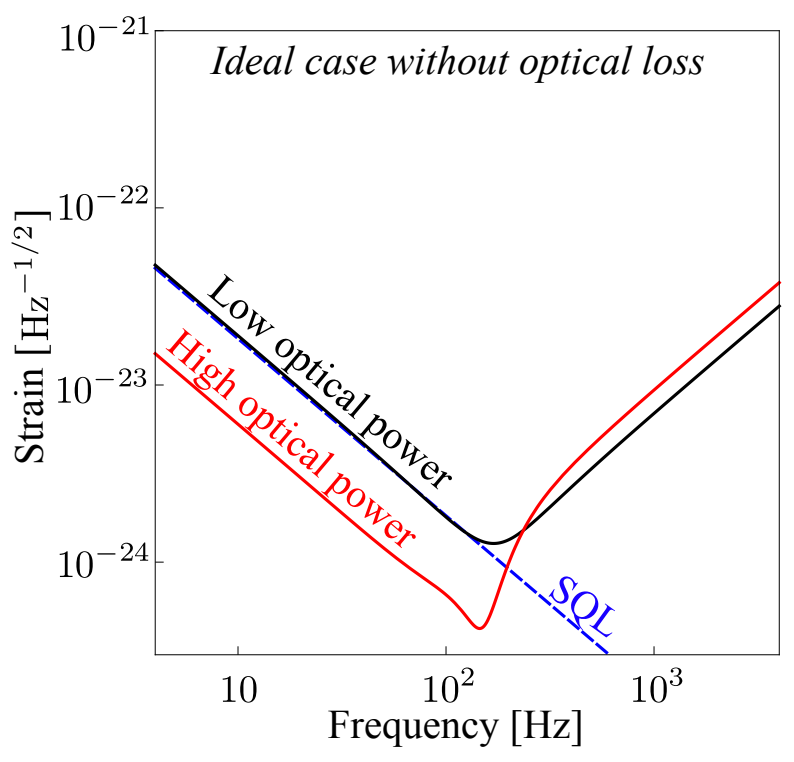

Figure 13: Noise spectrum for the speed-meter configuration with two different levels of circulating optical power.

The details of this scheme have been presented in [25], in particular the inputoutput relation which will be used in the numerical optimization. At this moment, we just show the resulting quantum-noise spectrum for this scheme:

$$
S_{h}(\Omega)=\frac{\left(\tan \zeta-\mathcal{K}_{\mathrm{sm}}\right)^{2}+1}{2 \mathcal{K}_{\mathrm{sm}}(\Omega)} h_{\mathrm{SQL}}^{2}(\Omega)
$$

with

$$
\mathcal{K}_{\mathrm{sm}}(\Omega)=\frac{16 \omega_{0} \gamma P_{c}}{m c L\left[\left(\Omega^{2}-\omega_{s}^{2}\right)^{2}+\gamma^{2} \Omega^{2}\right]} .
$$

Because $\mathcal{K}_{\text {sm }}$ has a flat frequency response, by properly choosing the homodyne angle $\zeta$, we can remove the low-frequency radiation pressure noise, and the sensitivity is only limited by the amount of optical power that we have. This noise spectrum is shown in Figure 13. The low-frequency spectrum has the same slope as the standard quantum limit, which is a unique feature of speed meter. When the optical power is high enough, we can surpass the standard quantum limit.

One important characteristic frequency for this type of speed meter is the sloshing frequency $\omega_{s}$, and it is defined as

$$
\omega_{s}=\frac{c}{2} \sqrt{\frac{T_{s}}{L L_{s}}}
$$

where $T_{s}$ is the power transmissivity for the front mirror of the sloshing cavity and $L_{s}$ is the cavity length. To achieve a speed response in the detection band, this sloshing frequency needs to be around $100 \mathrm{~Hz}$. For a $4 \mathrm{~km}$ arm cavity $-L=4000 \mathrm{~m}$ and $100 \mathrm{~m}$ sloshing cavity $-L_{s}=100 \mathrm{~m}$, it requires the transmittance of the sloshing mirror to be

$$
T_{s} \approx 30 \mathrm{ppm}
$$


This puts a rather tight constraint on the optical loss of the sloshing cavity. To release such a constraint on the optical loss, we can use the fact that $\omega_{s}$ only depends on the ratio between the transmissivity of the sloshing mirror and the cavity length and we can therefore increase the cavity length.

In addition, it seems that no filter cavity is needed for speed meter configuration, as the radiation pressure noise at low frequencies is canceled. However, such a cancellation is achieved by choosing the homodyne detection angle

$$
\zeta=\left.\arctan \mathcal{K}_{\mathrm{sm}}\right|_{\Omega \rightarrow 0},
$$

and a high optical power means a large $\mathcal{K}_{\mathrm{sm}}$ and therefore $\zeta$ deviates from 0 (the phase quadrature), decreasing sensitivity at high frequencies. With frequency-dependent squeezing, we can reduce $\mathcal{K}_{\text {sm }}$, or equivalently, $\zeta$, at low frequencies, which allows us to enhance the high-frequency sensitivity. Similarly, the frequency dependent readout allows us to cancel the low-frequency radiation pressure noise without sacrificing the high-frequency sensitivity by rotating the readout angle to the phase quadrature at high frequencies.

\subsection{Multiple Carrier Fields}
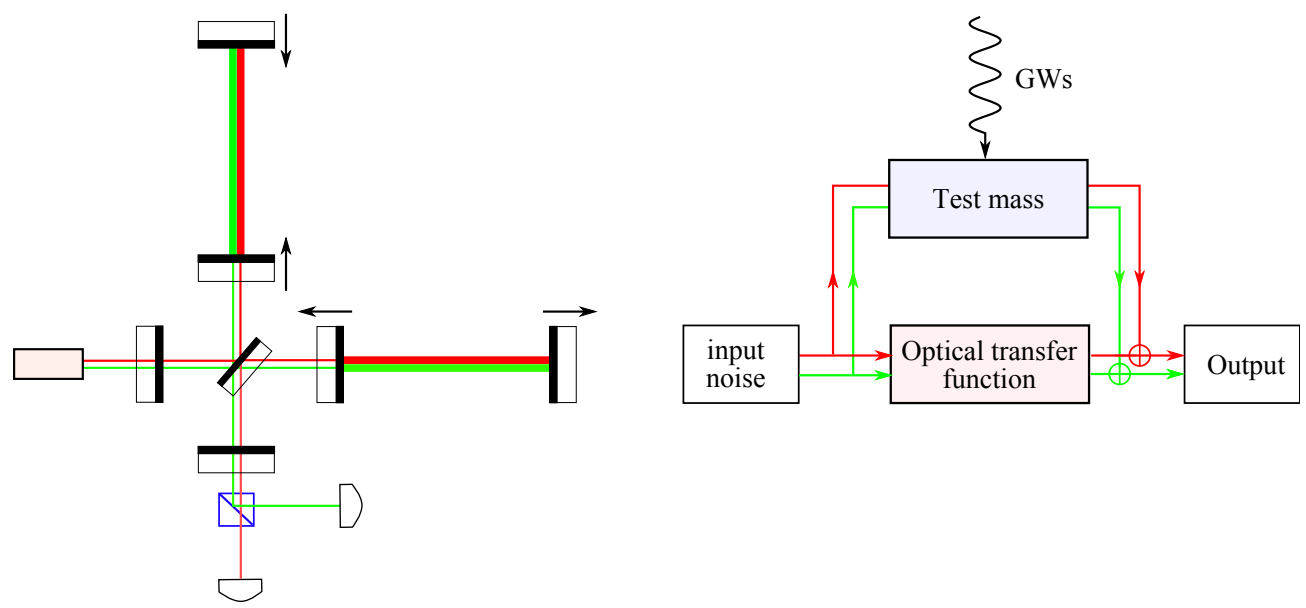

Figure 14: Schematics showing the dual-carrier scheme (left) and its block diagram (right).

In this section, we will introduce the multiple carrier scheme, and in particular, we will focus on the dual-carrier case as shown schematically in Figure 14. The additional carrier field provides us with another readout channel. As these two fields can have a very large frequency separation, we can, in principle, design the optics in such a way that they have different optical power and see different detuning and bandwidth. In addition, they can be independently measured at the output. This allows us to gain a lot flexibilities and effectively provides multiple interferometers but within the same set of optics.

These two optical fields are not completely independent, and they are coupled to each other as both act on the test masses and sense the test-mass motion (shown pictorially by the block diagram in Figure 14). More explicitly, we can look at the input-output relation for this scheme in the simple case when both fields are tuned 
Quantum Limits of Interferometer Topologies for Gravitational Radiation Detection 18

(in the SRC):

$$
\begin{aligned}
{\left[\begin{array}{c}
b_{1}^{(A)} \\
b_{2}^{(A)} \\
b_{1}^{(B)} \\
b_{2}^{(B)}
\end{array}\right]=} & {\left[\begin{array}{cccc}
1 & 0 & 0 & 0 \\
-\mathcal{K}_{A} & 1 & -\sqrt{\mathcal{K}_{A} \mathcal{K}_{B}} & 0 \\
0 & 0 & 1 & 0 \\
-\sqrt{\mathcal{K}_{A} \mathcal{K}_{B}} & 0 & -\mathcal{K}_{B} & 1
\end{array}\right]\left[\begin{array}{l}
a_{1}^{(A)} \\
a_{2}^{(A)} \\
a_{1}^{(B)} \\
a_{2}^{(B)}
\end{array}\right] } \\
+ & {\left[\begin{array}{c}
0 \\
\sqrt{2 \mathcal{K}_{A}} \\
0 \\
\sqrt{2 \mathcal{K}_{B}}
\end{array}\right] \frac{h}{h_{\mathrm{SQL}}}, }
\end{aligned}
$$

where we have ignored the uninteresting phase factor $e^{i \phi}$ and we have introduced

$$
\mathcal{K}_{A}=\frac{16 \omega_{0}^{(A)} \gamma_{A} P_{c}^{(A)}}{m L c \Omega^{2}\left(\Omega^{2}+\gamma_{A}^{2}\right)}, \quad \mathcal{K}_{B}=\frac{16 \omega_{0}^{(B)} \gamma_{B} P_{c}^{(B)}}{m L c \Omega^{2}\left(\Omega^{2}+\gamma_{B}^{2}\right)} .
$$

The term $-\sqrt{\mathcal{K}_{A} \mathcal{K}_{B}}$ in the transfer function matrix indicates the coupling between these two optical fields, and it comes from the fact that the radiation-pressure noise from the first one is sensed by the second one and vise versa.

As mentioned earlier, because the frequency separation between these two fields is much lager than the detection band, they can be measured independently and give two outputs $b_{\zeta}^{(A)}$ and $b_{\zeta}^{(B)}$ :

$$
b_{\zeta}^{(A)}=b_{1}^{(A)} \sin \zeta_{A}+b_{2}^{(A)} \cos \zeta_{A}, \quad b_{\zeta}^{(B)}=b_{1}^{(B)} \sin \zeta_{B}+b_{2}^{(B)} \cos \zeta_{B}
$$

To achieve the optimal sensitivity, we need to combine them with "optimal" filters $C_{A}(\Omega)$ and $C_{B}(\Omega)$, obtaining

$$
b_{\zeta}^{\text {tot }}(\Omega)=C_{A}(\Omega) b_{\zeta}^{(A)}(\Omega)+C_{B}(\Omega) b_{\zeta}^{(B)}(\Omega) .
$$

In Ref. [28], the authors have shown the procedure for obtaining the optimal sensitivity and the associated optimal filters in the general case with multiple carrier fields. Given the input-output relation: $\boldsymbol{b}=\mathbf{M} \boldsymbol{a}+\boldsymbol{v} h$-a simplified vector form of Equation 33, the noise spectrum that gives the optimal sensitivity is:

$$
S_{h}(\Omega)=\left[\boldsymbol{v}^{\dagger} \mathbf{M}_{\mathrm{hd}}^{\dagger}\left(\mathbf{M}_{\mathrm{hd}} \mathbf{M} \mathbf{M}^{\dagger} \mathbf{M}_{\mathrm{hd}}^{\dagger}\right)^{-1} \mathbf{M}_{\mathrm{hd}} \boldsymbol{v}\right]^{-1},
$$

where we have defined:

$$
\boldsymbol{M}_{\mathrm{hd}}=\left[\begin{array}{cccc}
\sin \zeta_{A} & \cos \zeta_{A} & 0 & 0 \\
0 & 0 & \sin \zeta_{B} & \cos \zeta_{B}
\end{array}\right] .
$$

This result is used for our numerical optimization in Section 4.

\subsection{Local Readout}

Here we will discuss a special case of the multiple-carrier scheme - the local-readout scheme, as shown schematically in Figure 15. In this scheme, the second carrier field is only resonant in the power-recycling cavity and is anti-resonant in the arm cavity (barely enters the arm cavity). Why we single this scheme out of the general dualcarrier scheme and give it a special name is more or less due to a historic reason. This scheme was first proposed in [28] and was motivated by trying to enhance the low-frequency sensitivity of a detuned signal-recycling interferometer, which is not as good as the tuned signal-recycling due to the optical-spring effect. The name "local 

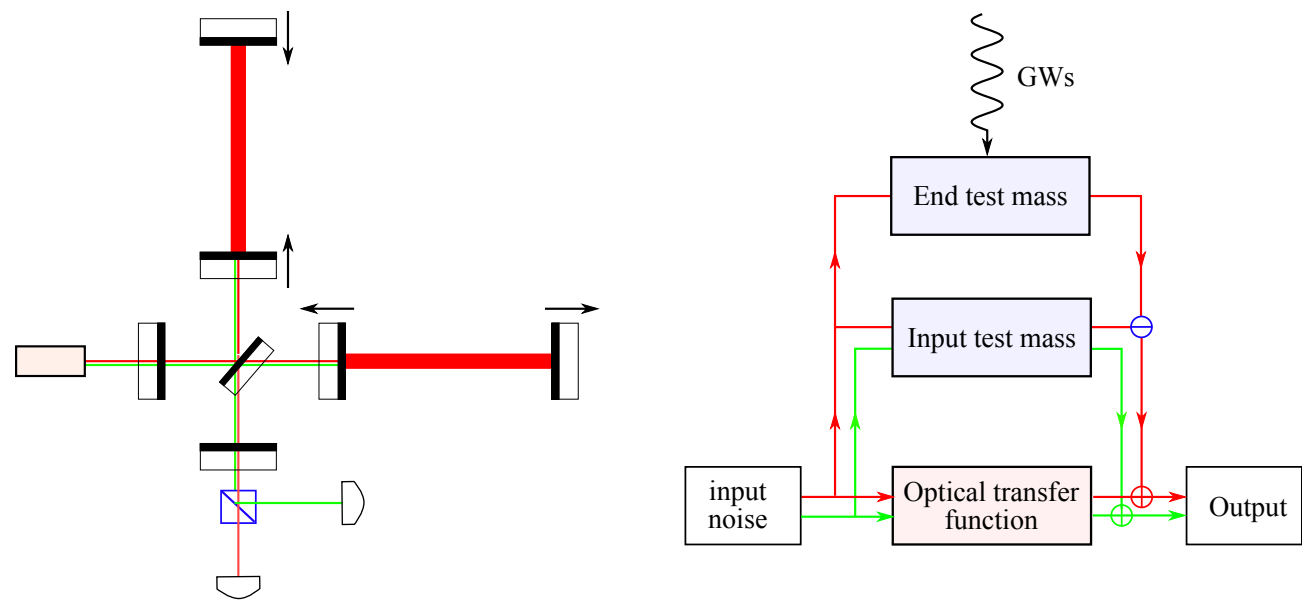

Figure 15: Schematics showing the local-readout topology (left) and the corresponding block diagram (right).

readout" originates from the fact that the second carrier field only measures the motion of the input test mass (ITM) which is local in the proper frame of the beam splitter and does not contain the gravitational-wave signal. One might ask: "how can we recover the detector sensitivity if the second carrier measures something that does not contain the signal?" Interestingly, even though no signal is measured by the second carrier, it measures the radiation-pressure noise of ITM introduced by the first carrier which has a much higher optical power due to the amplification of the arm cavity, as shown schematically by the block diagram of Figure 15. By combining the outputs of two carriers optimally, we can cancel some part of the radiation-pressure noise and enhance the sensitivity - the local-readout scheme can therefore be viewed as a noise-cancellation scheme. The cancellation efficiency is only limited by the radiationpressure noise of the second carrier field.

To evaluate the sensitivity for this scheme rigorously, one has to treat the input test mass (ITM) and end test mass (ETM) individually, instead of combining them into an single effective mass as we did for those schemes mentioned earlier. One can read [28] for details. 
Quantum Limits of Interferometer Topologies for Gravitational Radiation Detection20

\section{Numerical Optimization}

To arrive at the optimum sensitivity (as defined by Equation 1, our cost function), we use a simplex numerical optimization to vary the optical parameters for each of the previously described topologies. For this optimization, we also take into account the various classical noise sources (to be distinguished from the quantum noise). These noise sources are described in Appendix B.
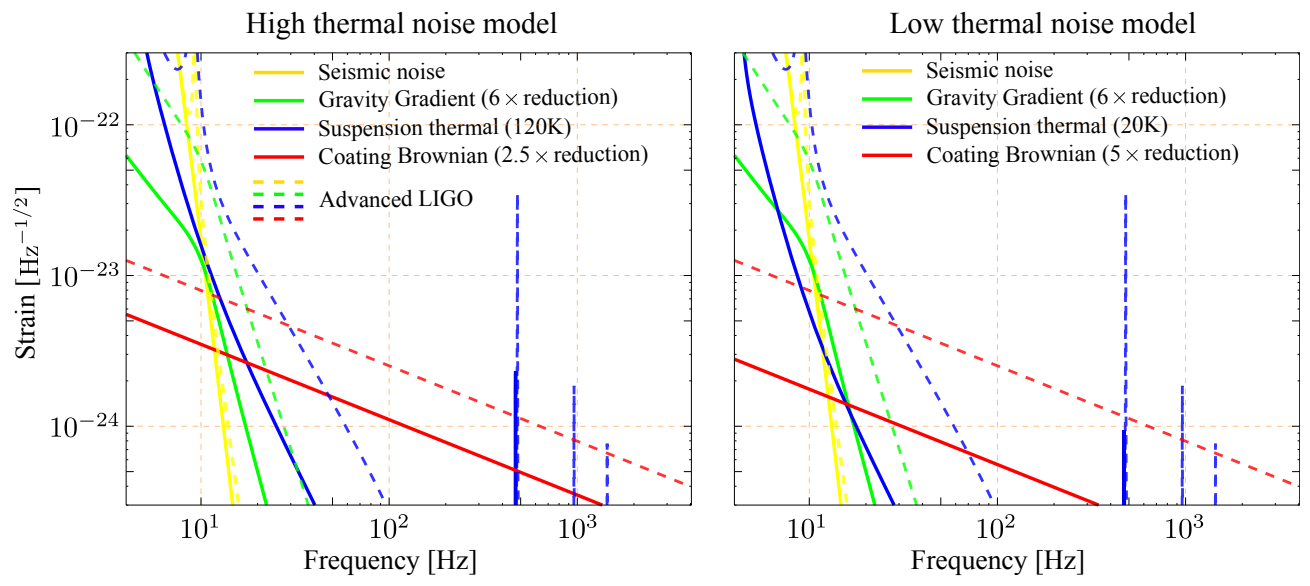

Figure 16: Spectra of the high classical noise model (left) and the low classical noise model (right).

\subsection{Cost Function}

The final optimization result critically depends on the cost function. In the literature, optimizations have been carried out by using a cost function that is source-orientedtrying to maximize the signal-to-noise ratio for particular astrophysical sources. Here we apply a rather different cost function, as shown in Eq. 1, that tries to maximize the broadband improvement over aLIGO.

\subsection{Optimization results}

For the optimization, we separate the configurations into two groups: (i) the frequencydependent squeezing (input filtering) group, in which we consider adding input filter cavities to those configurations mentioned in Section 3; (ii) the variational-readout (output filtering) group, in which we consider adding output filter cavities. Note that for those multiple-carrier schemes, e.g., the local-readout scheme, the number of filter cavities is equal to the number of carrier fields, and the number of optimization parameters therefore increases proportionally. In real implementations, we might specifically design one filter cavity that is able to simultaneously filter several carrier fields with different filtering parameters, and we can then reduce the number of optics.

4.2.1. Total noise spectrum The optimization result for the high classical noise model was shown at the very beginning (cf. Figure 1). Notice that, in that plot, we did not show the dual-carrier scheme with both carrier fields resonant in the arm cavities, and 
only showed the local-readout scheme in which only one carrier is resonant in the arm cavity. This is due to the interesting fact that when we fix the total power of the two carriers, the optimal power for one carrier turns out to be zero - this simply recovers the single-carrier case. Admittedly, this is due to the specific cost function and the thermal-noise model that we have chosen. In general, it is not clear that this would be optimal.

The optimization result for the low classical noise model was shown in Figure 2. It is clear that the general features are identical to the input-filtering one. The only prominent difference comes from the low-frequency sensitivities. This is attributable to the susceptibility to loss of the frequency dependent readout scheme, as mentioned early in Appendix C. Again, we can see that the speed meter and the local-readout scheme both allow significant improvements at low frequencies.

In Appendix A, we have listed the optimal values for the different parameters.
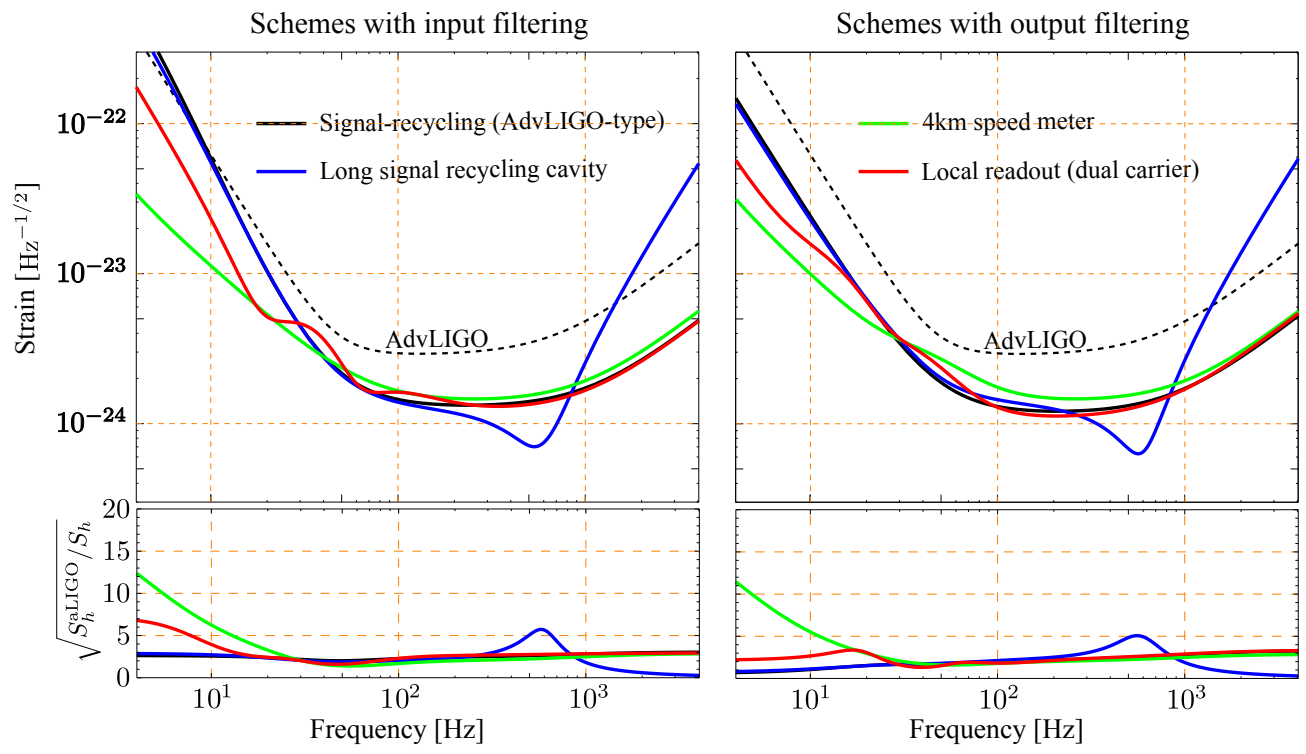

Figure 17: Plot showing the spectra for the quantum noise that contributes to the total noise shown in Figure 1.

4.2.2. Quantum noise contribution To compare the quantum noise contribution to the total noise spectrum, we show only the quantum noise spectrum in Figure 17 and Figure 18. It is clear that only at low frequencies do these schemes differ from each other distinctively. The low-frequency classical noise masks any difference. Therefore, unless significant changes can be made to reduce the low-frequency thermal noise, a sound reasoning - for choosing one advanced configuration over the other as a candidate for upgrade - should be based on the additional complexity involved, as different schemes do not perform drastically different after taking into account the thermal noise of the suspensions and mirrors. 
Quantum Limits of Interferometer Topologies for Gravitational Radiation Detection22
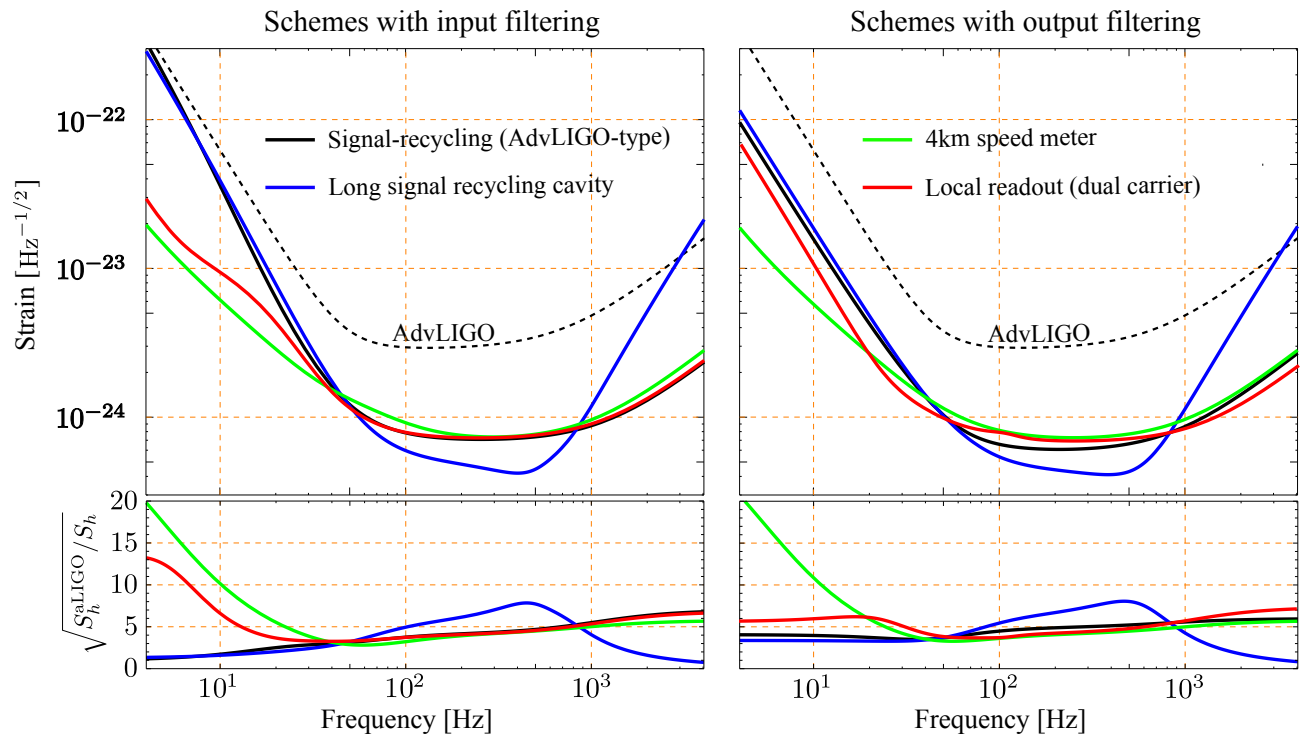

Figure 18: Spectra for the quantum noise shown in Figure 2.

\section{Future Studies}

In the current study, we only cover a few topologies among those that have been proposed in the literature. To proceed, one approach is to further expand the list of configurations, but this is a rather daunting task given the huge number of possible combinations. An alternative that we shall apply in the future is viewing optical and mechanical components as linear filters, and seeking the answer to the following question: "What is the optimal filter that we should place in between the test mass and the photodetector such that a specific cost function is minimized or if we know the signal waveform?" Similar techniques are employed in the design of electronic circuits and optimal search algorithms for finding signals in noisy data. The only subtlety is that we are dealing with quantum and classical fluctuations - there are certain constraints on the filters that one must apply in order to preserve the quantum coherence, especially in cases of amplitude filtering.

To be concrete, let us look at the structure of the detection process more carefully. The test mass, which contains the GW signal, turns ingoing optical fields into outgoing fields which in turn are detected by the photodetector. In between the test mass and the photodetector, the most generic filter we can apply is a four-port filter, as illustrated in Figure 19. The transfer functions of such a four-port filter- $T_{a}(\Omega), T_{b}(\Omega)$, $R_{a}(\Omega)$ and $R_{b}(\Omega) \|$ - are not independent and need to satisfy the Stokes relation due to energy conservation and time-reversal symmetry. Specifically, if we separate their amplitude and phase as follows:

$$
\begin{aligned}
T_{a}(\Omega) & =\left|T_{a}(\Omega)\right| e^{i \phi_{a}(\Omega)}, \quad T_{b}(\Omega)=\left|T_{a}(\Omega)\right| e^{i \phi_{b}(\Omega)}, \\
R_{a}(\Omega) & =\left|R_{a}(\Omega)\right| e^{i \varphi_{a}(\Omega)}, \quad R_{b}(\Omega)=\left|R_{b}(\Omega)\right| e^{i \varphi_{b}(\Omega)},
\end{aligned}
$$

|| For simplicity, here we use the sideband picture instead of quadrature, otherwise these transfer functions will be transfer matrices. 


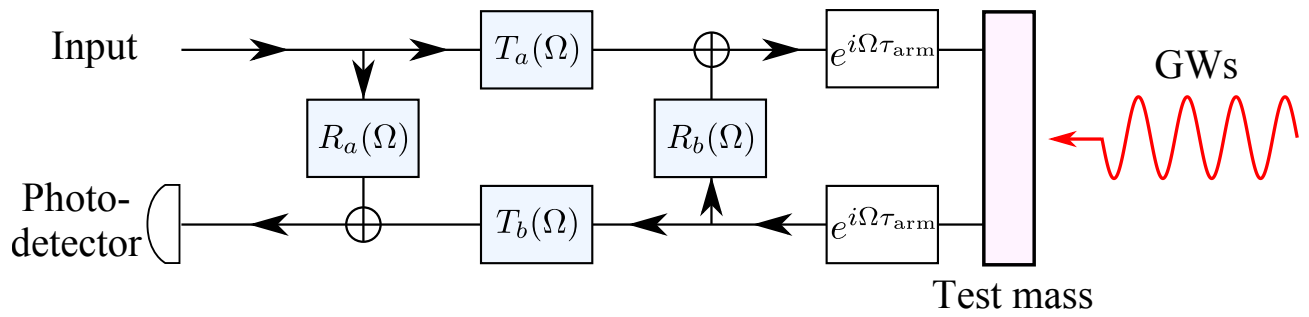

Figure 19: Schematics illustrating the generic four-port filter that can be applied in between the test mass and the photodetector. Here we are considering one sideband frequency $\Omega$; $\tau_{\text {arm }}=L / c$ is the time delay by the interferometer arm.

the Stokes relation dictates the following constraints:

$$
\begin{aligned}
& \left|T_{a}(\Omega)\right|=\left|T_{b}(\Omega)\right|, \quad\left|R_{a}(\Omega)\right|=\left|R_{b}(\Omega)\right|, \quad|T(\Omega)|^{2}+|R(\Omega)|^{2}=1, \\
& e^{i \phi_{a}(\Omega)}=e^{i \phi_{b}(\Omega)}, \quad e^{i \varphi_{a}(\Omega)+i \varphi_{b}(\Omega)}=-e^{2 i \phi_{a}(\Omega)} .
\end{aligned}
$$

In order to obtain the optimal four-port filter given a certain cost function, we can either (i) parameterize those transfer functions in terms of zeros and poles and optimize them - this requires a mapping between zeros and poles to the physical setup, which is highly nontrivial, or (ii) insert a number of cavities and optimize the parameters - this is more transparent in terms of finding out the physical scheme. As a first attack, we will apply the latter approach, as illustrated in Figure 20. Not only do we consider input filtering $T_{1}(\Omega)$ and output filtering $T_{2}(\Omega)$, we also include the intra-cavity filtering $T_{3}(\Omega)$ and $T_{4}(\Omega)$ - the filters sit inside the signal-recycling cavity (the sloshing cavity in the speed-meter configuration is one special example of the intra-cavity filtering). These filters are different cascades of cavities that can either have fixed mirrors (the passive cavity) or a movable end mirror (the opto-mechanical cavity). The usual passive optical cavity only allows us to create a frequencydependent phase shift on the sidebands, or equivalently, frequency-dependent rotation of the amplitude and phase quadratures. By adding control light and allowing the end mirror to be movable, we can also create frequency-dependent amplitude modulation, similar to the ponderomotive squeezer proposed in [39]. Recently such active cavities with opto-mechanical interactions have triggered interesting discussion within the GW community, as it allows us to filter the audio-band signal with table-top scale setups. However, to realize it experimentally, the mirror thermal noise needs to be low enough such that the quantum coherence shall not be destroyed. This probably requires cryogenic temperatures which is somewhat challenging to realize. In future numerical optimization, we will study the influence of thermal noise in the opto-mechanical cavity on the sensitivity.

\section{Conclusions}

We have optimized the quantum noise spectrum for a few different interferometer configurations that are candidates for the $3^{\text {rd }}$ generation LIGO. In particular, we have considered the frequency dependent squeezing (input filtering) and frequency dependent readout (output filtering); introducing additional filter cavities either at the input or the output ports. Limited by thermal noise at low frequencies, the difference among these configurations is not very prominent. This leads us to the 
Quantum Limits of Interferometer Topologies for Gravitational Radiation Detection24
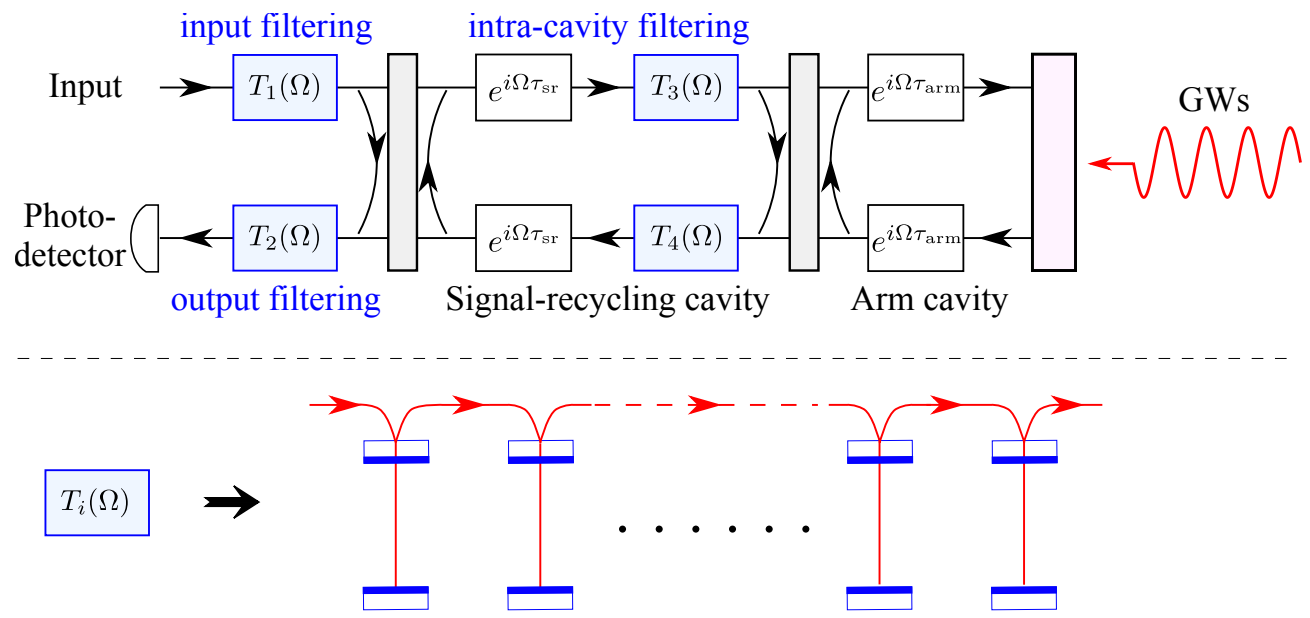

Figure 20: Schematics illustrating the scheme that we will numerically optimize (top). Each of these transfer functions corresponds to a cascade of (opto-mechanical) cavities in series (bottom).

conclusion that adding one input filter cavity to Advanced LIGO seems to be the most feasible approach for upgrading in the near term, due to its simplicity compared with other schemes. If the low-frequency thermal noise can be reduced in the future, the speed meter and the multiple-carrier scheme can provide significant low-frequency enhancement of the sensitivity. This extra enhancement will, for some low enough thermal noise, be enough to compensate for the extra complexity.

\section{Acknowledgments}

We would like to thank our colleagues in the LIGO Scientific Collaboration's Advanced Interferometer Configurations working group for fruitful discussions. R. X. A. is supported by NSF grant PHY-0757058. H. M., H. Y., and Y. C. are supported by NSF grants PHY-0555406, PHY-0653653, PHY-0601459, PHY-0956189, PHY-1068881, as well as the David and Barbara Groce startup fund at Caltech.

\section{References}

[1] BP Abbott, R. Abbott, R. Adhikari, P. Ajith, B. Allen, G. Allen, RS Amin, SB Anderson, WG Anderson, MA Arain, et al. LIGO: the laser interferometer gravitational-wave observatory. Reports on Progress in Physics, 72:076901, 2009.

[2] Hartmut Grote. The GEO 600 status. Classical and Quantum Gravity, 27(8):084003, 2010.

[3] T Accadia, F Acernese, F Antonucci, P Astone, G Ballardin, F Barone, M Barsuglia, A Basti, Th S Bauer, M Bebronne, M G Beker, A Belletoile, S Birindelli, M Bitossi, M A Bizouard, M Blom, F Bondu, L Bonelli, R Bonnand, V Boschi, L Bosi, B Bouhou, S Braccini, C Bradaschia, M Branchesi, T Briant, A Brillet, V Brisson, R Budzyński, T Bulik, H J Bulten, D Buskulic, C Buy, G Cagnoli, E Calloni, B Canuel, F Carbognani, F Cavalier, R Cavalieri, G Cella, E Cesarini, O Chaibi, E Chassande Mottin, A Chincarini, F Cleva, E Coccia, P-F Cohadon, C N Colacino, J Colas, A Colla, M Colombini, A Corsi, J-P Coulon, E Cuoco, S D'Antonio, V Dattilo, M Davier, R Day, R De Rosa, G Debreczeni, W Del Pozzo, M del Prete, L Di Fiore, A Di Lieto, M Di Paolo Emilio, A Di Virgilio, A Dietz, M Drago, V Fafone, I Ferrante, F Fidecaro, I Fiori, R Flaminio, L A Forte, JD Fournier, J Franc, S Frasca, F Frasconi, M Galimberti, L Gammaitoni, F Garufi, M E 
Quantum Limits of Interferometer Topologies for Gravitational Radiation Detection25

Gáspár, G Gemme, E Genin, A Gennai, A Giazotto, R Gouaty, M Granata, C Greverie, G M Guidi, J-F Hayau, A Heidmann, H Heitmann, P Hello, D Huet, P Jaranowski, I Kowalska, A Królak, N Leroy, N Letendre, T G F Li, N Liguori, M Lorenzini, V Loriette, G Losurdo, E Majorana, I Maksimovic, N Man, M Mantovani, F Marchesoni, F Marion, J Marque, F Martelli, A Masserot, C Michel, L Milano, Y Minenkov, M Mohan, N Morgado, A Morgia, S Mosca, V Moscatelli, B Mours, F Nocera, G Pagliaroli, L Palladino, C Palomba, F Paoletti, M Parisi, A Pasqualetti, R Passaquieti, D Passuello, G Persichetti, M Pichot, F Piergiovanni, M Pietka, L Pinard, R Poggiani, M Prato, G A Prodi, M Punturo, P Puppo, D S Rabeling, I Rácz, P Rapagnani, V Re, T Regimbau, F Ricci, F Robinet, A Rocchi, L Rolland, R Romano, D Rosińska, P Ruggi, B Sassolas, D Sentenac, L Sperandio, R Sturani, B Swinkels, M Tacca, L Taffarello, A Toncelli, M Tonelli, O Torre, E Tournefier, F Travasso, G Vajente, J F J van den Brand, C Van Den Broeck, S van der Putten, M Vasuth, M Vavoulidis, G Vedovato, D Verkindt, F Vetrano, A Viceré, J-Y Vinet, S Vitale, H Vocca, R L Ward, M Was, M Yvert, and J-P Zendri. Status of the Virgo project. Classical and Quantum Gravity, 28(11):114002, 2011.

[4] K Arai, R Takahashi, D Tatsumi, K Izumi, Y Wakabayashi, H Ishizaki, M Fukushima, T Yamazaki, M-K Fujimoto, A Takamori, K Tsubono, R DeSalvo, A Bertolini, S Márka, V Sannibale, the TAMA Collaboration, T Uchiyama, O Miyakawa, S Miyoki, K Agatsuma, T Saito, M Ohashi, K Kuroda, I Nakatani, S Telada, K Yamamoto, T Tomaru, T Suzuki, T Haruyama, N Sato, A Yamamoto, T Shintomi, the CLIO Collaboration, and The LCGT Collaboration. Status of japanese gravitational wave detectors. Classical and Quantum Gravity, 26(20):204020, 2009.

[5] J Abadie, B P Abbott, R Abbott, M Abernathy, T Accadia, F Acernese, C Adams, R Adhikari, P Ajith, B Allen, G Allen, E Amador Ceron, R S Amin, S B Anderson, W G Anderson, F Antonucci, S Aoudia, M A Arain, M Araya, M Aronsson, K G Arun, Y Aso, S Aston, P Astone, D E Atkinson, P Aufmuth, C Aulbert, S Babak, P Baker, G Ballardin, S Ballmer, D Barker, S Barnum, F Barone, B Barr, P Barriga, L Barsotti, M Barsuglia, M A Barton, I Bartos, R Bassiri, M Bastarrika, J Bauchrowitz, Th S Bauer, B Behnke, M G Beker, M Benacquista, A Bertolini, J Betzwieser, N Beveridge, P T Beyersdorf, S Bigotta, I A Bilenko, G Billingsley, J Birch, S Birindelli, R Biswas, M Bitossi, M A Bizouard, E Black, J K Blackburn, L Blackburn, D Blair, B Bland, M Blom, A Blomberg, C Boccara, O Bock, T P Bodiya, R Bondarescu, F Bondu, L Bonelli, R Bork, M Born, S Bose, L Bosi, M Boyle, S Braccini, C Bradaschia, P R Brady, V B Braginsky, J E Brau, J Breyer, D O Bridges, A Brillet, M Brinkmann, V Brisson, M Britzger, A F Brooks, D A Brown, R Budzyński, T Bulik, H J Bulten, A Buonanno, J Burguet-Castell, O Burmeister, D Buskulic, R L Byer, L Cadonati, G Cagnoli, E Calloni, J B Camp, E Campagna, P Campsie, J Cannizzo, K C Cannon, B Canuel, J Cao, C Capano, F Carbognani, S Caride, S Caudill, M Cavaglià, F Cavalier, R Cavalieri, G Cella, C Cepeda, E Cesarini, T Chalermsongsak, E Chalkley, P Charlton, E Chassande Mottin, S Chelkowski, Y Chen, A Chincarini, N Christensen, S S Y Chua, C T Y Chung, D Clark, J Clark, J H Clayton, F Cleva, E Coccia, C N Colacino, J Colas, A Colla, M Colombini, R Conte, D Cook, T R Corbitt, C Corda, N Cornish, A Corsi, C A Costa, J P Coulon, D Coward, D C Coyne, J D E Creighton, T D Creighton, A M Cruise, R M Culter, A Cumming, L Cunningham, E Cuoco, K Dahl, S L Danilishin, R Dannenberg, S D'Antonio, K Danzmann, A Dari, K Das, V Dattilo, B Daudert, M Davier, G Davies, A Davis, E J Daw, R Day, T Dayanga, R De Rosa, D DeBra, J Degallaix, M del Prete, V Dergachev, R DeRosa, R DeSalvo, P Devanka, S Dhurandhar, L Di Fiore, A Di Lieto, I Di Palma, M Di Paolo Emilio, A Di Virgilio, M Díaz, A Dietz, F Donovan, K L Dooley, E E Doomes, S Dorsher, E S D Douglas, M Drago, R W P Drever, J C Driggers, J Dueck, J C Dumas, T Eberle, M Edgar, M Edwards, A Effler, P Ehrens, R Engel, T Etzel, M Evans, T Evans, V Fafone, S Fairhurst, Y Fan, B F Farr, D Fazi, H Fehrmann, D Feldbaum, I Ferrante, F Fidecaro, L S Finn, I Fiori, R Flaminio, M Flanigan, K Flasch, S Foley, C Forrest, E Forsi, N Fotopoulos, J D Fournier, J Franc, S Frasca, F Frasconi, M Frede, M Frei, Z Frei, A Freise, R Frey, T T Fricke, D Friedrich, P Fritschel, V V Frolov, P Fulda, M Fyffe, L Gammaitoni, J A Garofoli, F Garufi, G Gemme, E Genin, A Gennai, I Gholami, S Ghosh, J A Giaime, S Giampanis, K D Giardina, A Giazotto, C Gill, E Goetz, L M Goggin, G González, M L Gorodetsky, S Goßler, R Gouaty, C Graef, M Granata, A Grant, S Gras, C Gray, R J S Greenhalgh, A M Gretarsson, C Greverie, R Grosso, H Grote, S Grunewald, G M Guidi, E K Gustafson, R Gustafson, B Hage, P Hall, J M Hallam, D Hammer, G Hammond, J Hanks, C Hanna, J Hanson, J Harms, G M Harry, I W Harry, E D Harstad, K Haughian, K Hayama, J Heefner, H Heitmann, P Hello, I S Heng, A Heptonstall, M Hewitson, S Hild, E Hirose, D Hoak, K A Hodge, K Holt, D J Hosken, 
Quantum Limits of Interferometer Topologies for Gravitational Radiation Detection26

J Hough, E Howell, D Hoyland, D Huet, B Hughey, S Husa, S H Huttner, T Huynh-Dinh, D R Ingram, R Inta, T Isogai, A Ivanov, P Jaranowski, W W Johnson, D I Jones, G Jones, R Jones, L Ju, P Kalmus, V Kalogera, S Kandhasamy, J Kanner, E Katsavounidis, K Kawabe, S Kawamura, F Kawazoe, W Kells, D G Keppel, A Khalaidovski, F Y Khalili, E A Khazanov, C Kim, H Kim, P J King, D L Kinzel, J S Kissel, S Klimenko, V Kondrashov, R Kopparapu, S Koranda, I Kowalska, D Kozak, T Krause, V Kringel, S Krishnamurthy, B Krishnan, A Królak, G Kuehn, J Kullman, R Kumar, P Kwee, M Landry, M Lang, B Lantz, N Lastzka, A Lazzarini, P Leaci, J Leong, I Leonor, N Leroy, N Letendre, J Li, T G F Li, H Lin, P E Lindquist, N A Lockerbie, D Lodhia, M Lorenzini, V Loriette, M Lormand, G Losurdo, P Lu, J Luan, M Lubinski, A Lucianetti, H Lück, A Lundgren, B Machenschalk, M MacInnis, J M Mackowski, M Mageswaran, K Mailand, E Majorana, C Mak, N Man, I Mandel, V Mandic, M Mantovani, F Marchesoni, F Marion, S Márka, Z Márka, E Maros, J Marque, F Martelli, I W Martin, R M Martin, J N Marx, K Mason, A Masserot, F Matichard, L Matone, R A Matzner, N Mavalvala, R McCarthy, D E McClelland, S C McGuire, G McIntyre, G McIvor, D J A McKechan, G Meadors, M Mehmet, T Meier, A Melatos, A C Melissinos, G Mendell, D F Menéndez, R A Mercer, L Merill, S Meshkov, C Messenger, M S Meyer, H Miao, C Michel, L Milano, J Miller, Y Minenkov, Y Mino, S Mitra, V P Mitrofanov, G Mitselmakher, R Mittleman, B Moe, M Mohan, S D Mohanty, S R P Mohapatra, D Moraru, J Moreau, G Moreno, N Morgado, A Morgia, T Morioka, K Mors, S Mosca, V Moscatelli, K Mossavi, B Mours, C MowLowry, G Mueller, S Mukherjee, A Mullavey, H Müller-Ebhardt, J Munch, P G Murray, T Nash, R Nawrodt, J Nelson, I Neri, G Newton, A Nishizawa, F Nocera, D Nolting, E Ochsner, J O'Dell, G H Ogin, R G Oldenburg, B O'Reilly, R O'Shaughnessy, C Osthelder, D J Ottaway, R S Ottens, H Overmier, B J Owen, A Page, G Pagliaroli, L Palladino, C Palomba, Y Pan, C Pankow, F Paoletti, M A Papa, S Pardi, M Pareja, M Parisi, A Pasqualetti, R Passaquieti, D Passuello, P Patel, M Pedraza, L Pekowsky, S Penn, C Peralta, A Perreca, G Persichetti, M Pichot, M Pickenpack, F Piergiovanni, M Pietka, L Pinard, I M Pinto, M Pitkin, H J Pletsch, M V Plissi, R Poggiani, F Postiglione, M Prato, V Predoi, L R Price, M Prijatelj, M Principe, S Privitera, R Prix, G A Prodi, L Prokhorov, O Puncken, M Punturo, P Puppo, V Quetschke, F J Raab, O Rabaste, D S Rabeling, T Radke, H Radkins, P Raffai, M Rakhmanov, B Rankins, P Rapagnani, V Raymond, V Re, C M Reed, T Reed, T Regimbau, S Reid, D H Reitze, F Ricci, R Riesen, K Riles, P Roberts, N A Robertson, F Robinet, C Robinson, E L Robinson, A Rocchi, S Roddy, C Röver, S Rogstad, L Rolland, J Rollins, J D Romano, R Romano, J H Romie, D Rosińska, S Rowan, A Rüdiger, P Ruggi, K Ryan, S Sakata, M Sakosky, F Salemi, L Sammut, L Sancho de la Jordana, V Sandberg, V Sannibale, L Santamaría, G Santostasi, S Saraf, B Sassolas, B S Sathyaprakash, S Sato, M Satterthwaite, P R Saulson, R Savage, R Schilling, R Schnabel, R Schofield, B Schulz, B F Schutz, P Schwinberg, J Scott, S M Scott, A C Searle, F Seifert, D Sellers, A S Sengupta, D Sentenac, A Sergeev, D A Shaddock, B Shapiro, P Shawhan, D H Shoemaker, A Sibley, X Siemens, D Sigg, A Singer, A M Sintes, G Skelton, B J J Slagmolen, J Slutsky, J R Smith, M R Smith, N D Smith, K Somiya, B Sorazu, F C Speirits, A J Stein, L C Stein, S Steinlechner, S Steplewski, A Stochino, R Stone, K A Strain, S Strigin, A Stroeer, R Sturani, A L Stuver, T Z Summerscales, M Sung, S Susmithan, P J Sutton, B Swinkels, D Talukder, D B Tanner, S P Tarabrin, J R Taylor, R Taylor, P Thomas, K A Thorne, K S Thorne, E Thrane, A Thüring, C Titsler, K V Tokmakov, A Toncelli, M Tonelli, C Torres, C I Torrie, E Tournefier, F Travasso, G Traylor, M Trias, J Trummer, K Tseng, D Ugolini, K Urbanek, H Vahlbruch, B Vaishnav, G Vajente, M Vallisneri, J F J van den Brand, C Van Den Broeck, $\mathrm{S}$ van der Putten, M V van der Sluys, A A van Veggel, S Vass, R Vaulin, M Vavoulidis, A Vecchio, G Vedovato, J Veitch, P J Veitch, C Veltkamp, D Verkindt, F Vetrano, A Viceré, A Villar, J-Y Vinet, H Vocca, C Vorvick, S P Vyachanin, S J Waldman, L Wallace, A Wanner, R L Ward, M Was, P Wei, M Weinert, A J Weinstein, R Weiss, L Wen, S Wen, P Wessels, M West, T Westphal, K Wette, J T Whelan, S E Whitcomb, D J White, B F Whiting, C Wilkinson, P A Willems, L Williams, B Willke, L Winkelmann, W Winkler, C C Wipf, A G Wiseman, G Woan, R Wooley, J Worden, I Yakushin, H Yamamoto, K Yamamoto, D Yeaton-Massey, S Yoshida, P P Yu, M Yvert, M Zanolin, L Zhang, Z Zhang, C Zhao, N Zotov, M E Zucker, J Zweizig, The LIGO Scientific Collaboration, the Virgo Collaboration, and K Belczynski. Predictions for the rates of compact binary coalescences observable by ground-based gravitational-wave detectors. Classical and Quantum Gravity, 27(17):173001, 2010.

[6] Advanced LIGO. https://www.advancedligo.mit.edu/.

[7] The Virgo Collaboration. Advanced Virgo technical design report. Technical report, April 2012.

[8] Christopf Affeldt, Jerome Degallaix, Andreas Freise, Hartmut Grote, Martin Hewitson, Stefan 
Hild, Jonathan Leong, Mirko Prijatelj, Kenneth A Strain, Benno Willke, Holger Wittel, and Karsten Danzmann. The upgrade of GEO600. arXiv.org, gr-qc, April 2010.

[9] K. Somiya and for the LCGT Collaboration. Detector configuration of LCGT - the Japanese cryogenic gravitational-wave detector. ArXiv e-prints, November 2011.

[10] Rana X. Adhikari. Gravitational radiation detection with laser interferometry. Rev. Mod. Phys., 86(1):121-151, February 2014.

[11] Thomas Corbitt and Nergis Mavalvala. Review: Quantum noise in gravitational-wave interferometers. Journal of Optics B: Quantum and Semiclassical Optics, 6(8):S675, 2004.

[12] Yanbei Chen. Macroscopic quantum mechanics: theory and experimental concepts of optomechanics. Journal of Physics B: Atomic, Molecular and Optical Physics, 46(10):104001, 2013.

[13] R. Schnabel, N. Mavalvala, D. E. McClelland, and P. K. Lam. Quantum metrology for gravitational wave astronomy. Nature Communications, 1, November 2010.

[14] David E McClelland, Nergis Mavalvala, Y Chen, and Roman Schnabel. Advanced interferometry, quantum optics and optomechanics in gravitational wave detectors. Laser 8 Photonics Reviews, 5(5):677-696, 2011.

[15] J Abadie, B P Abbott, R Abbott, M Abernathy, T Accadia, F Acernese, C Adams, R Adhikari, P Ajith, B Allen, G Allen, E Amador Ceron, R S Amin, S B Anderson, W G Anderson, F Antonucci, S Aoudia, M A Arain, M Araya, M Aronsson, K G Arun, Y Aso, S Aston, P Astone, D E Atkinson, P Aufmuth, C Aulbert, S Babak, P Baker, G Ballardin, S Ballmer, D Barker, S Barnum, F Barone, B Barr, P Barriga, L Barsotti, M Barsuglia, M A Barton, I Bartos, R Bassiri, M Bastarrika, J Bauchrowitz, Th S Bauer, B Behnke, M G Beker, M Benacquista, A Bertolini, J Betzwieser, N Beveridge, P T Beyersdorf, S Bigotta, I A Bilenko, G Billingsley, J Birch, S Birindelli, R Biswas, M Bitossi, M A Bizouard, E Black, J K Blackburn, L Blackburn, D Blair, B Bland, M Blom, A Blomberg, C Boccara, O Bock, T P Bodiya, R Bondarescu, F Bondu, L Bonelli, R Bork, M Born, S Bose, L Bosi, M Boyle, S Braccini, C Bradaschia, P R Brady, V B Braginsky, J E Brau, J Breyer, D O Bridges, A Brillet, M Brinkmann, V Brisson, M Britzger, A F Brooks, D A Brown, R Budzyński, T Bulik, H J Bulten, A Buonanno, J Burguet-Castell, O Burmeister, D Buskulic, R L Byer, L Cadonati, G Cagnoli, E Calloni, J B Camp, E Campagna, P Campsie, J Cannizzo, K C Cannon, B Canuel, J Cao, C Capano, F Carbognani, S Caride, S Caudill, M Cavaglià, F Cavalier, R Cavalieri, G Cella, C Cepeda, E Cesarini, T Chalermsongsak, E Chalkley, P Charlton, E Chassande Mottin, S Chelkowski, Y Chen, A Chincarini, N Christensen, S S Y Chua, C T Y Chung, D Clark, J Clark, J H Clayton, F Cleva, E Coccia, C N Colacino, J Colas, A Colla, M Colombini, R Conte, D Cook, T R Corbitt, C Corda, N Cornish, A Corsi, C A Costa, J P Coulon, D Coward, D C Coyne, J D E Creighton, T D Creighton, A M Cruise, R M Culter, A Cumming, L Cunningham, E Cuoco, K Dahl, S L Danilishin, R Dannenberg, S D'Antonio, K Danzmann, A Dari, K Das, V Dattilo, B Daudert, M Davier, G Davies, A Davis, E J Daw, R Day, T Dayanga, R De Rosa, D DeBra, J Degallaix, M del Prete, V Dergachev, R DeRosa, R DeSalvo, P Devanka, S Dhurandhar, L Di Fiore, A Di Lieto, I Di Palma, M Di Paolo Emilio, A Di Virgilio, M Díaz, A Dietz, F Donovan, K L Dooley, E E Doomes, S Dorsher, E S D Douglas, M Drago, R W P Drever, J C Driggers, J Dueck, J C Dumas, T Eberle, M Edgar, M Edwards, A Effler, P Ehrens, R Engel, T Etzel, M Evans, T Evans, V Fafone, S Fairhurst, Y Fan, B F Farr, D Fazi, H Fehrmann, D Feldbaum, I Ferrante, F Fidecaro, L S Finn, I Fiori, R Flaminio, M Flanigan, K Flasch, S Foley, C Forrest, E Forsi, N Fotopoulos, J D Fournier, J Franc, S Frasca, F Frasconi, M Frede, M Frei, Z Frei, A Freise, R Frey, T T Fricke, D Friedrich, P Fritschel, V V Frolov, P Fulda, M Fyffe, L Gammaitoni, J A Garofoli, F Garufi, G Gemme, E Genin, A Gennai, I Gholami, S Ghosh, J A Giaime, S Giampanis, K D Giardina, A Giazotto, C Gill, E Goetz, L M Goggin, G González, M L Gorodetsky, S Goßler, R Gouaty, C Graef, M Granata, A Grant, S Gras, C Gray, R J S Greenhalgh, A M Gretarsson, C Greverie, R Grosso, H Grote, S Grunewald, G M Guidi, E K Gustafson, R Gustafson, B Hage, P Hall, J M Hallam, D Hammer, G Hammond, J Hanks, C Hanna, J Hanson, J Harms, G M Harry, I W Harry, E D Harstad, K Haughian, K Hayama, J Heefner, H Heitmann, P Hello, I S Heng, A Heptonstall, M Hewitson, S Hild, E Hirose, D Hoak, K A Hodge, K Holt, D J Hosken, J Hough, E Howell, D Hoyland, D Huet, B Hughey, S Husa, S H Huttner, T Huynh-Dinh, D R Ingram, R Inta, T Isogai, A Ivanov, P Jaranowski, W W Johnson, D I Jones, G Jones, R Jones, L Ju, P Kalmus, V Kalogera, S Kandhasamy, J Kanner, E Katsavounidis, K Kawabe, S Kawamura, F Kawazoe, W Kells, D G Keppel, A Khalaidovski, F Y Khalili, E A Khazanov, C Kim, H Kim, P J King, D L Kinzel, J S Kissel, S Klimenko, V Kondrashov, R Kopparapu, S Koranda, I Kowalska, D Kozak, T Krause, V Kringel, S Krishnamurthy, B Krishnan, 
Quantum Limits of Interferometer Topologies for Gravitational Radiation Detection28

A Królak, G Kuehn, J Kullman, R Kumar, P Kwee, M Landry, M Lang, B Lantz, N Lastzka, A Lazzarini, P Leaci, J Leong, I Leonor, N Leroy, N Letendre, J Li, T G F Li, H Lin, P E Lindquist, N A Lockerbie, D Lodhia, M Lorenzini, V Loriette, M Lormand, G Losurdo, P Lu, J Luan, M Lubinski, A Lucianetti, H Lück, A Lundgren, B Machenschalk, M MacInnis, J M Mackowski, M Mageswaran, K Mailand, E Majorana, C Mak, N Man, I Mandel, V Mandic, M Mantovani, F Marchesoni, F Marion, S Márka, Z Márka, E Maros, J Marque, F Martelli, I W Martin, R M Martin, J N Marx, K Mason, A Masserot, F Matichard, L Matone, R A Matzner, N Mavalvala, R McCarthy, D E McClelland, S C McGuire, G McIntyre, G McIvor, D J A McKechan, G Meadors, M Mehmet, T Meier, A Melatos, A C Melissinos, G Mendell, D F Menéndez, R A Mercer, L Merill, S Meshkov, C Messenger, M S Meyer, H Miao, C Michel, L Milano, J Miller, Y Minenkov, Y Mino, S Mitra, V P Mitrofanov, G Mitselmakher, R Mittleman, B Moe, M Mohan, S D Mohanty, S R P Mohapatra, D Moraru, J Moreau, G Moreno, N Morgado, A Morgia, T Morioka, K Mors, S Mosca, V Moscatelli, K Mossavi, B Mours, C MowLowry, G Mueller, S Mukherjee, A Mullavey, H Müller-Ebhardt, J Munch, P G Murray, T Nash, R Nawrodt, J Nelson, I Neri, G Newton, A Nishizawa, F Nocera, D Nolting, E Ochsner, J O'Dell, G H Ogin, R G Oldenburg, B O'Reilly, R O'Shaughnessy, C Osthelder, D J Ottaway, R S Ottens, H Overmier, B J Owen, A Page, G Pagliaroli, L Palladino, C Palomba, Y Pan, C Pankow, F Paoletti, M A Papa, S Pardi, M Pareja, M Parisi, A Pasqualetti, R Passaquieti, D Passuello, P Patel, M Pedraza, L Pekowsky, S Penn, C Peralta, A Perreca, G Persichetti, M Pichot, M Pickenpack, F Piergiovanni, M Pietka, L Pinard, I M Pinto, M Pitkin, H J Pletsch, M V Plissi, R Poggiani, F Postiglione, M Prato, V Predoi, L R Price, M Prijatelj, M Principe, S Privitera, R Prix, G A Prodi, L Prokhorov, O Puncken, M Punturo, P Puppo, V Quetschke, F J Raab, O Rabaste, D S Rabeling, T Radke, H Radkins, P Raffai, M Rakhmanov, B Rankins, P Rapagnani, V Raymond, V Re, C M Reed, T Reed, T Regimbau, S Reid, D H Reitze, F Ricci, R Riesen, K Riles, P Roberts, N A Robertson, F Robinet, C Robinson, E L Robinson, A Rocchi, S Roddy, C Röver, S Rogstad, L Rolland, J Rollins, J D Romano, R Romano, J H Romie, D Rosińska, S Rowan, A Rüdiger, P Ruggi, K Ryan, S Sakata, M Sakosky, F Salemi, L Sammut, L Sancho de la Jordana, V Sandberg, V Sannibale, L Santamaría, G Santostasi, S Saraf, B Sassolas, B S Sathyaprakash, S Sato, M Satterthwaite, P R Saulson, R Savage, R Schilling, R Schnabel, R Schofield, B Schulz, B F Schutz, P Schwinberg, J Scott, S M Scott, A C Searle, F Seifert, D Sellers, A S Sengupta, D Sentenac, A Sergeev, D A Shaddock, B Shapiro, P Shawhan, D H Shoemaker, A Sibley, X Siemens, D Sigg, A Singer, A M Sintes, G Skelton, B J J Slagmolen, J Slutsky, J R Smith, M R Smith, N D Smith, K Somiya, B Sorazu, F C Speirits, A J Stein, L C Stein, S Steinlechner, S Steplewski, A Stochino, R Stone, K A Strain, S Strigin, A Stroeer, R Sturani, A L Stuver, T Z Summerscales, M Sung, S Susmithan, P J Sutton, B Swinkels, D Talukder, D B Tanner, S P Tarabrin, J R Taylor, R Taylor, P Thomas, K A Thorne, K S Thorne, E Thrane, A Thüring, C Titsler, K V Tokmakov, A Toncelli, M Tonelli, C Torres, C I Torrie, E Tournefier, F Travasso, G Traylor, M Trias, J Trummer, K Tseng, D Ugolini, K Urbanek, H Vahlbruch, B Vaishnav, G Vajente, M Vallisneri, J F J van den Brand, C Van Den Broeck, S van der Putten, M V van der Sluys, A A van Veggel, S Vass, R Vaulin, M Vavoulidis, A Vecchio, G Vedovato, J Veitch, P J Veitch, C Veltkamp, D Verkindt, F Vetrano, A Viceré, A Villar, J-Y Vinet, H Vocca, C Vorvick, S P Vyachanin, S J Waldman, L Wallace, A Wanner, R L Ward, M Was, P Wei, M Weinert, A J Weinstein, $\mathrm{R}$ Weiss, L Wen, S Wen, P Wessels, M West, T Westphal, K Wette, J T Whelan, S E Whitcomb, D J White, B F Whiting, C Wilkinson, P A Willems, L Williams, B Willke, L Winkelmann, W Winkler, C C Wipf, A G Wiseman, G Woan, R Wooley, J Worden, I Yakushin, H Yamamoto, K Yamamoto, D Yeaton-Massey, S Yoshida, P P Yu, M Yvert, M Zanolin, L Zhang, Z Zhang, C Zhao, N Zotov, M E Zucker, J Zweizig, The LIGO Scientific Collaboration, the Virgo Collaboration, and K Belczynski. Squeezed light injection in a gravitational wave detector. in prep., 2012.

[16] H. J. Kimble, Y. Levin, A. B. Matsko, K. S. Thorne, and S. P. Vyatchanin. Conversion of conventional gravitational-wave interferometers into quantum nondemolition interferometers by modifying their input and/or output optics. Phys. Rev. D, 65:022002, 2001.

[17] J. Harms et al. Squeezed-input, optical-spring, signal-recycled gravitational-wave detectors. Phys. Rev. D, 68:042001, 2003.

[18] Thomas Corbitt, Nergis Mavalvala, and Stan Whitcomb. Optical cavities as amplitude filters for squeezed fields. Phys. Rev. D, 70:022002, Jul 2004.

[19] F. Ya. Khalili. Optimal configurations of filter cavity in future gravitational-wave detectors. Phys. Rev. D, 81:122002, Jun 2010.

[20] M. Evans, L. Barsotti, P. Kwee, J. Harms, and H. Miao. Realistic filter cavities for advanced 
Quantum Limits of Interferometer Topologies for Gravitational Radiation Detection29

gravitational wave detectors. Phys. Rev. D, 88:022002, Jul 2013.

[21] S. P. Vyatchanin and E. A. Zubova. Quantum variation measurement of a force. Physics Letters A, 201(4):269-274, May 1995.

[22] F. Ya. Khalili. Quantum variational measurement in the next generation gravitational-wave detectors. Phys. Rev. D, 76:102002, 2007.

[23] F. Ya. Khalili and Yu. Levin. Speed meter as a quantum nondemolition measuring device for force. Phys. Rev. D, 54:004735, 1996.

[24] P. Purdue. Analysis of a quantum nondemolition speed-meter interferometer. Physical Review $D, 66(2): 022001$, June 2002.

[25] P. Purdue and Y. Chen. Practical speed meter designs for quantum nondemolition gravitationalwave interferometers. Physical Review D, 66(12):122004, December 2002.

[26] S. L. Danilishin. Sensitivity limitations in optical speed meter topology of gravitational-wave antennas. Phys. Rev. D, 69:102003, 2004.

[27] Y. Chen, S.L. Danilishin, F.Ya. Khalili, and H. Müller-Ebhardt. QND measurements for future gravitational-wave detectors. General Relativity and Gravitation, 43:671-694, 2011.

[28] Henning Rehbein, Helge Müller-Ebhardt, Kentaro Somiya, Chao Li, Roman Schnabel, Karsten Danzmann, and Yanbei Chen. Local readout enhancement for detuned signal-recycling interferometers. Phys. Rev. D, 76:062002, Sep 2007.

[29] Einstein Telescope Science Team. Einstein gravitational wave Telescope conceptual design study, 2011.

[30] Stefan L. Danilishin and Farid Ya. Khalili. Quantum measurement theory in gravitational-wave detectors. Living Reviews in Relativity, 15(5), 2012.

[31] V. B. Braginsky and F. Y. Khalili. Quantum Measurement. Cambridge University Press, 1999.

[32] David E McClelland. An overview of recycling in laser interferometric gravitation wave detectors. Australian Journal of Physics, 48:953-970, 1995.

[33] A. Buonanno and Y. Chen. Scaling law in signal recycled laser-interferometer gravitational-wave detectors. Phys. Rev. D, 67:062002, 2003.

[34] A Thüring, R Schnabel, H Lück, and K Danzmann. Detuned twin-signal-recycling for ultrahighprecision interferometers. Optics letters, 32:985-987, 2007.

[35] Christian Gräf, André Thüring, Henning Vahlbruch, Karsten Danzmann, and Roman Schnabel. Length sensing and control of a michelson interferometer with power recycling and twin signal recycling cavities. Optics express, 21:5287-99, 2013.

[36] Y. Chen. Sagnac interferometer as a speed-meter-type, quantum-nondemolition gravitationalwave detector. Physical Review D, 67(12):122004, June 2003.

[37] Peter T. Beyersdorf, M. M. Fejer, and R. L. Byer. Polarization sagnac interferometer with postmodulation for gravitational-wave detection. Opt. Lett., 24(16):1112-1114, Aug 1999.

[38] Andrew R. Wade, Kirk McKenzie, Yanbei Chen, Daniel A. Shaddock, Jong H. Chow, and David E. McClelland. Polarization speed meter for gravitational-wave detection. Phys. Rev. $D$, 86:062001, Sep 2012.

[39] Thomas Corbitt, Yanbei Chen, Farid Khalili, David Ottaway, Sergey Vyatchanin, Stan Whitcomb, and Nergis Mavalvala. Squeezed-state source using radiation-pressure-induced rigidity. Phys. Rev. A, 73(2):023801-, February 2006.

[40] N. A. Robertson, G. Cagnoli, D. R. M. Crooks, E. Elliffe, J. E. Faller, P. Fritschel, S. Goßler, A. Grant, A. Heptonstall, J. Hough, H. Lück, R. Mittleman, M. Perreur-Lloyd, M. V. Plissi, S. Rowan, D. H. Shoemaker, P. H. Sneddon, K. A. Strain, C. I. Torrie, H. Ward, and P. Willems. Quadruple suspension design for Advanced LIGO. Classical and Quantum Gravity, 19:4043-4058, August 2002.

[41] A V Cumming, A S Bell, L Barsotti, M A Barton, G Cagnoli, D Cook, L Cunningham, M Evans, G D Hammond, G M Harry, A Heptonstall, J Hough, R Jones, R Kumar, R Mittleman, N A Robertson, S Rowan, B Shapiro, K A Strain, K Tokmakov, C Torrie, and A A van Veggel. Design and development of the Advanced LIGO monolithic fused silica suspension. Classical and Quantum Gravity, 29(3):035003, 2012.

[42] N. A. Robertson for the LSC. Seismic isolation and suspension systems for Advanced LIGO. Proceedings of SPIE, 5500:81, 2004.

[43] Peter R. Saulson. Terrestrial gravitational noise on a gravitational wave antenna. Phys. Rev. D, 30:732-736, Aug 1984 .

[44] J. C. Driggers, J. Harms, and R. X. Adhikari. Subtraction of Newtonian noise using optimized sensor arrays. Physical Review D, 86(10):102001, November 2012.

[45] Gregory M Harry, Matthew R Abernathy, Andres E Becerra-Toledo, Helena Armandula, Eric Black, Kate Dooley, Matt Eichenfield, Chinyere Nwabugwu, Akira Villar, D R M Crooks, Gianpietro Cagnoli, Jim Hough, Colin R How, Ian MacLaren, Peter Murray, Stuart 
Quantum Limits of Interferometer Topologies for Gravitational Radiation Detection30

Reid, Sheila Rowan, Peter H Sneddon, Martin M Fejer, Roger Route, Steven D Penn, Patrick Ganau, Jean-Marie Mackowski, Christophe Michel, Laurent Pinard, and Alban Remillieux. Titania-doped tantala/silica coatings for gravitational-wave detection. Classical and Quantum Gravity, 24(2):405, 2007.

[46] T. Hong, H. Yang, E. K. Gustafson, R. X. Adhikari, and Y. Chen. Brownian thermal noise in multilayer coated mirrors. Physical Review D, 87(8):082001, April 2013.

[47] T. Isogai, J. Miller, P. Kwee, L. Barsotti, and M. Evans. Losses in long-storage-time optical cavities. ArXiv e-prints, October 2013.

[48] A.E. Siegman. Lasers. University Science Books, 1986.

[49] Hiro Yamamoto. LIGO I mirror scattering loss by microroughness. LIGO Document, T070082, Apr 2007.

[50] Christopher J. Walsh, Achim J. Leistner, and Bozenko F. Oreb. Power spectral density analysis of optical substrates for gravitational-wave interferometry. Appl. Opt., 38(22):4790-4801, Aug 1999.

\section{Appendix A. Optimal Parameters}

Optimal parameters for the configurations with frequency dependent squeeze angle in the high thermal noise model are listed in Table A1. The nominal parameters common among different configurations are: $m=50 \mathrm{~kg}, T_{\mathrm{PRM}}=0.03, T_{\mathrm{ITM}}=0.01$ and maximal input power is equal to $125 \mathrm{~W}$, which corresponds to around $1 \mathrm{MW}$ circulating in the arm cavity. For the squeezed light, we have assumed $10 \mathrm{~dB}$ squeezing with $5 \%$ injection loss, arising from the lossy optics between the squeezer and the input mirror of the filter cavity. For the speed meter, the length of the sloshing cavity is $4 \mathrm{~km}$ and the power transmittance for the sloshing mirror is equal to $700 \mathrm{ppm}$. Note that all the configurations are tuned, as a broadband sensitivity is preferred, given the particular cost function that we have chosen.

\begin{tabular}{|c|c|c|c|c|c|}
\hline Configurations & $P_{0}(\mathrm{~W})$ & $T_{\mathrm{sr}}$ & $T_{f}(\mathrm{ppm})$ & $\Delta_{f}(\mathrm{~Hz})$ & $\mathrm{FOM}$ \\
\hline Signal-recycling & 125.0 & 0.12 & 249.2 & -28.5 & 206.5 \\
\hline Long signal recycling & 125.0 & 0.1 & 179.2 & -21.7 & 186.5 \\
\hline Speed meter & 125.0 & 0.07 & 334.1 & -24.2 & 202.4 \\
\hline Local readout (carrier A) & 125.0 & 0.14 & 164.0 & -19.0 & 207.2 \\
Local readout (carrier B) & 46.2 & 0.0013 & 145.0 & -13.6 & - \\
\hline
\end{tabular}

Table A1: Optimal parameters for different configurations with frequency-dependent squeezing in the high thermal noise model. Here $P_{0}$ is the input optical power, $T_{\mathrm{sr}}$ is the power transmittance of the signal recycling mirror, $T_{f}$ is the power transmittance for the front mirror of the filter cavity, $\Delta_{f}$ is the detune frequency of the filter cavity, and figure of merit $(\mathrm{FOM})$ is equal to $10^{6} / \mathcal{C}$ with $\mathcal{C}$ the value of the cost function defined in Eq. $1-$ the larger the figure of merit is, the better the broadband sensitivity is.

The optimal parameters in the high thermal noise model for different configurations with frequency dependent (variational) readout quadrature are listed in the Table A2. The common parameters are the same as those for the frequency dependent squeezing. The transmissivity of the sloshing mirror for the speed meter is equal to $900 \mathrm{ppm}$.

The optimal parameters for different configurations with frequency dependent squeezing in the low thermal noise model are listed in the Table A3. The common parameters for different configurations are: $m=150 \mathrm{~kg}, T_{\mathrm{PRM}}=0.03, T_{\mathrm{ITM}}=0.01$ and maximal input power is equal to $500 \mathrm{~W}$, which corresponds to approximately $3 \mathrm{MW}$ intra cavity power. The specification for the squeezing is the same as the 
Quantum Limits of Interferometer Topologies for Gravitational Radiation Detection31

\begin{tabular}{|c|c|c|c|c|c|}
\hline Configurations & $P_{0}(\mathrm{~W})$ & $T_{\mathrm{sr}}$ & $T_{f}(\mathrm{ppm})$ & $\Delta_{f}(\mathrm{~Hz})$ & $\mathrm{FOM}$ \\
\hline Signal-recycling & 125.0 & 0.1 & 233.3 & -25.7 & 196.4 \\
\hline Long signal recycling & 125.0 & 0.10 & 231.6 & -25.5 & 179.3 \\
\hline Speed meter & 125.0 & 0.27 & 321.7 & -16.7 & 202.7 \\
\hline Local readout (carrier A) & 125.0 & 0.12 & 858.5 & -22.5 & 205.2 \\
Local readout (carrier B) & 38.0 & 0.001 & 858.1 & -21.9 & - \\
\hline
\end{tabular}

Table A2: Optimal parameters for different configurations with frequency dependent readout in the high thermal noise model.

low-noise model case - $10 \mathrm{~dB}$ squeezing with $5 \%$ injection loss. The optimal sloshing mirror transmittance for the speed meter is equal to $970 \mathrm{ppm}$.

\begin{tabular}{|c|c|c|c|c|c|}
\hline Configurations & $P_{0}(\mathrm{~W})$ & $T_{\mathrm{sr}}$ & $T_{f}(\mathrm{ppm})$ & $\Delta_{f}(\mathrm{~Hz})$ & $\mathrm{FOM}$ \\
\hline Signal-recycling & 500.0 & 0.12 & 256.9 & -31.4 & 259.9 \\
\hline Long signal recycling & 500.0 & 0.19 & 372.4 & -43.6 & 240.8 \\
\hline Speed meter & 500.0 & 0.28 & 335.4 & -20.1 & 260.2 \\
\hline Local readout (carrier A) & 500.0 & 0.092 & 234.1 & -22.1 & 267.9 \\
Local readout (carrier B) & 494.2 & 0.0001 & 135.8 & -49.0 & - \\
\hline
\end{tabular}

Table A3: Optimal parameters for different configurations with frequency-dependent squeezing in the low thermal noise model.

The optimal parameters for the low-noise model for different configurations with frequency dependent readout quadrature are listed in the Table A4. The optimal sloshing mirror transmittance for the speed meter is equal to 0.0013 .

\begin{tabular}{|c|c|c|c|c|c|}
\hline Configurations & $P_{0}(\mathrm{~W})$ & $T_{\mathrm{sr}}$ & $T_{f}(\mathrm{ppm})$ & $\Delta_{f}(\mathrm{~Hz})$ & $\mathrm{FOM}$ \\
\hline Signal-recycling & 500.0 & 0.087 & 247.5 & -27.4 & 249.3 \\
\hline Long signal recycling & 500.0 & 0.16 & 335.9 & -37.0 & 228.2 \\
\hline Speed meter & 500.0 & 0.16 & 516.5 & -18.4 & 257.5 \\
\hline Local readout (carrier A) & 424.0 & 0.097 & 245.2 & -25.9 & 257.6 \\
Local readout (carrier B) & 16.2 & 0.033 & 1000 & 14.1 & - \\
\hline
\end{tabular}

Table A4: Optimal parameters for different configurations with frequency dependent readout quadrature in the low thermal noise model.

\section{Appendix B. Other Noise Sources}

In addition to the quantum noise arising from the fluctuations in the ground state of the electromagnetic field, the sensitivity of the interferometers is also limited by Brownian thermal noise in the mirror suspensions [40,41], seismic vibrations propagating to the mirror [42], terrestrial gravitational fluctuations [43, 44], and Brownian thermal fluctuations of the mirror (and mirror coating) surface [45, 46]. In Table B1, we show the physical interferometer parameters used in the optimization. 


\begin{tabular}{|l|l|l|l|l|l|l|l|}
\hline Config & $M(\mathrm{~kg})$ & $\begin{array}{l}T_{\mathrm{mir}} \\
(\mathrm{K})\end{array}$ & $\begin{array}{l}T_{\text {sus }} \\
(\mathrm{K})\end{array}$ & $\begin{array}{l}w_{\text {beam }} \\
(\mathrm{cm})\end{array}$ & $\begin{array}{l}\phi_{\text {coat }}^{\text {high }} \\
10^{-5}\end{array}$ & $\begin{array}{l}\phi_{\text {coat }}^{\text {low }} \\
10^{-5}\end{array}$ & $N N_{\mathrm{FF}}$ \\
\hline aLIGO & 40 & 295 & 295 & 5.9 & 23 & 4.0 & - \\
\hline $\begin{array}{l}\text { High- } \\
\text { noise } \\
\text { model 1 }\end{array}$ & 50 & 295 & 120 & 14.6 & 23 & 4.0 & 6 \\
\hline $\begin{array}{l}\text { Low- } \\
\text { noise } \\
\text { model } 2\end{array}$ & 150 & 120 & 20 & 5.9 & 2.0 & 2.0 & 6 \\
\hline
\end{tabular}

Table B1: Physical parameters used for computing the non-quantum noise sources during the configuration optimization. Here $M$ is the mass of a single test mass; $T_{\operatorname{mir}}$ and $T_{\text {sus }}$ are the temperature of the mirrors (test masses) and the suspension wire, respectively; $w_{\text {beam }}$ is the average beam spot radius on the arm cavity mirrors; $\phi_{\text {coat }}^{\text {high }}$ and $\phi_{\text {coat }}^{\text {low }}$ are the mechanical loss angle for the high (low) refractive index materials, respectively; $N N_{\mathrm{FF}}$ is the Newtonian noise subtraction factor achieved by feed-forward cancellation.

\section{Appendix C. Optical loss and optimal filter cavity length}

It is essential to gain a full understanding - through experiments and numerical modeling - of how the optical loss scales with the cavity length, and this will determine the cavity length for achieving the optimal sensitivity. Here we will provide a qualitative estimate of the dependence of sensitivity on the optical loss and the cavity length, the connection between which is left for future work.

\section{Appendix C.1. Qualitative picture}

Given a filter cavity, the detector sensitivity, is affected by the total loss of the cavity $\mathcal{E}$ which is equal to the round-trip loss $\epsilon$ multiplied by the number of round trips $N \sim 1 / T_{f}$ with $T_{f}$ being the transmittance of the cavity input mirror (assuming a totally reflected end mirror), namely

$$
\mathcal{E} \approx \frac{\epsilon}{T_{f}} .
$$

In addition, since the filter cavity bandwidth $\gamma_{f}$ needs to be comparable to the detection bandwidth $\gamma$ of the interferometer in order to reduce the quantum noise, we require $\gamma_{f}=c T_{0} /\left(4 L_{f}\right) \approx \gamma$, where $L_{f}$ is the filter cavity length. It follows that

$$
T_{f} \approx \frac{4 \gamma L_{f}}{c} .
$$

Therefore, the total optical loss is given by:

$$
\mathcal{E} \approx \frac{c \epsilon}{4 \gamma L_{f}} \propto \frac{\epsilon}{L_{f}},
$$

which means that the total optical loss depends on the ratio between the round-trip loss and the filter cavity length.

If the optical loss were independent of the cavity length, the above scaling would imply that the longer the cavity, the smaller the total loss. However, this is usually not the case. Due to the roughness of the mirror surface, there will be an optical loss associated with scattering off the surface. One signifcant contribution comes from the "figure error" which corresponds to roughness with a spatial scale larger than 
a few millimeters; another is from micro-roughness due to small-scale imperfections which induce large-angle scattering. This scattering critically depends on the beam size which in turn depends on the cavity length [47]. To be more specific, the beam size $w_{f}$ for a confocal cavity $\uparrow$ is given by:

$$
w_{f}=\left(\frac{L_{f} \lambda}{2 \pi}\right)^{1 / 2}
$$

The dependence of scattering loss on the beam size is more involved. Here we only discuss the scattering associated with micro-roughness (the one from "figure error requires a numerical simulation and normally does not have a compact analytical expression).The corresponding scattering loss for an isotropic surface topology reads [49]:

$$
\epsilon=\left(\frac{4 \pi}{\lambda}\right)^{2} \int_{f_{s}^{\min }}^{f_{s}^{\max }} S_{1}\left(f_{s}\right) \mathrm{d} f_{s}
$$

Here $f_{s}$ has the units of $m^{-1}$ and is the spatial frequency; $f_{s}^{\min }$ is the minimum spatial frequency, or equivalently, the maximum length scale of the beam, and it is inversely proportional to the beam size:

$$
f_{s}^{\min } \approx\left(w_{f} / 2\right)^{-1}
$$

and the maximum spatial frequency $f_{s}^{\max }$ is taken to be $1 / \lambda$. The one dimensional scattering power spectral density $S_{1}\left(f_{s}\right)$ depends on specific optics that we use. For initial LIGO mirrors, the measured power spectral density approximately satisfies [50]:

$$
S_{1}\left(f_{s}\right)=7 \times 10^{-19} f_{s}^{-1.45} .
$$

In Figure C1, we show how the optical loss depends on the filter cavity length (numerically integrating the above integral). It can be approximated by the following power law:

$$
\left.\epsilon\right|_{\text {micro-roughness }} \approx 5.0 L_{f}^{0.23} .
$$

In this case, the total optical loss $\mathcal{E}$ [cf. Equation C.3] scales as $L_{f}^{-0.77}$. This seems to imply the longer the cavity the better. However, this ignores scattering loss from figure errors and also diffraction. In reality, to determine the optimal cavity length, we need to experimentally investigate the dependence of the round-trip optical loss on the cavity length.

\section{Appendix C.2. Detailed analysis}

Here we can provide a more detailed analysis to elaborate on the qualitative picture that we showed in the previous section concerning the magnitude of the loss. To account for the optical loss of filter cavities carefully, we need to inject vacuum fluctuations at every port of all optics ${ }^{+}$, summarizing the effect from scattering and absorption. The simple linear lossy cavity is illustrated in Figure $\mathrm{C} 2$, and we can derive its input-output relation from which we can determine quantitatively how the loss influences the quantum coherence of the squeezed light (for input filtering) or the output quadratures (for output filtering).

I A confocal cavity allows the minimal beam size on the mirror (see e.g. Chapter 19 in [48]).

+ Note that in the literature, normally one introduces a so-called lossy mirror to account for all the optical loss and assumes other mirrors are lossless. This works generally, but may fail when the optical path is complicated. Also there is some ambiguity in determining the loss for such a effective mirror. 


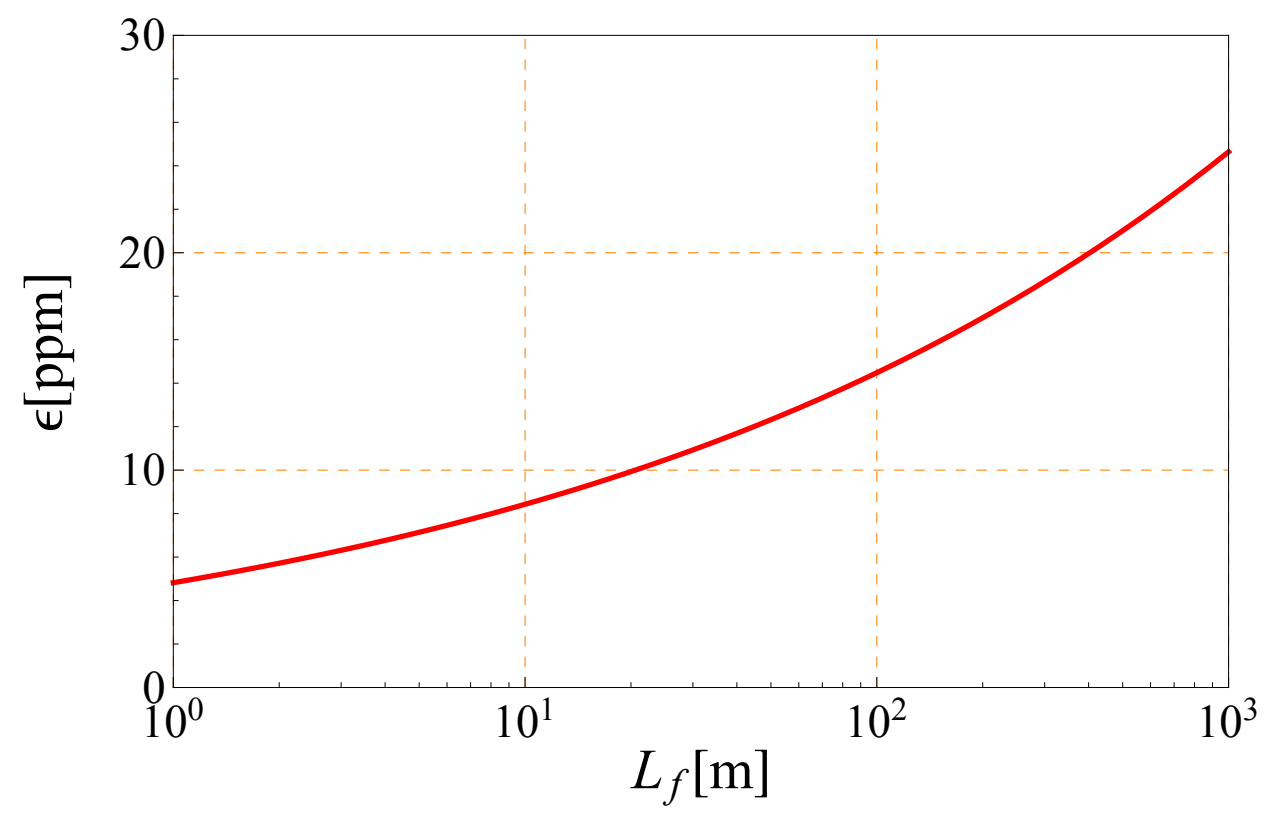

Figure C1: Dependence of scattering loss on the length of the filter cavity due to microroughness of the mirror surface. Figure errors at large spatial frequencies are neglected here.
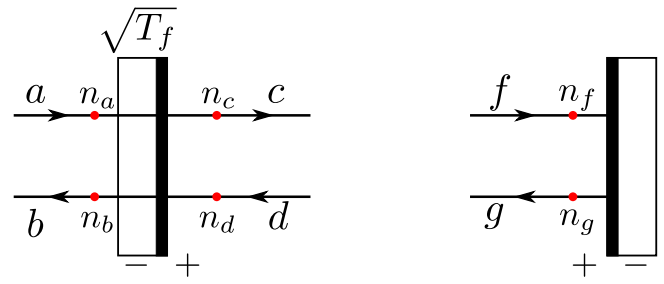

Figure C2: Figure illustrating the propagating fields in a single lossy filter cavity. The red dots are the lossy ports where vacuum fluctuations $n_{\alpha_{s}}$ that are uncorrelated with the main fields $\alpha_{s}$ enter $\left(\alpha_{s}=a, b, c, d, f, g\right)$ the optical paths.

To derive the input-output relation, we use the continuity condition of the fields, which goes as follows:

$$
\begin{aligned}
\boldsymbol{b}= & (1-\epsilon / 4)\left(-\sqrt{R_{f}} \boldsymbol{a}+\sqrt{T_{f}} \boldsymbol{d}\right)+\sqrt{(\epsilon / 4)(1-\epsilon / 4)}\left(-\sqrt{R_{f}} \boldsymbol{n}_{a}\right. \\
& \left.+\sqrt{T_{f}} \boldsymbol{n}_{d}\right)+\sqrt{\epsilon / 4} \boldsymbol{n}_{b} \\
\boldsymbol{c}= & (1-\epsilon / 4)\left(\sqrt{R_{f}} \boldsymbol{d}+\sqrt{T_{f}} \boldsymbol{a}\right)+\sqrt{(\epsilon / 4)(1-\epsilon / 4)}\left(\sqrt{R_{f}} \boldsymbol{n}_{d}\right. \\
& \left.+\sqrt{T_{f}} \boldsymbol{n}_{a}\right)+\sqrt{\epsilon / 4} \boldsymbol{n}_{c} \\
\boldsymbol{f}= & \mathbf{M}_{\mathrm{rot}} \boldsymbol{c} \\
\boldsymbol{g}= & (1-\epsilon / 4) \boldsymbol{f}+\sqrt{(\epsilon / 4)(1-\epsilon / 4)} \boldsymbol{n}_{f}+\sqrt{\epsilon / 4} \boldsymbol{n}_{g} \\
\boldsymbol{d}= & \mathbf{M}_{\mathrm{rot}} \boldsymbol{g}
\end{aligned}
$$

Here $\boldsymbol{\alpha}=\left(\alpha_{1}, \alpha_{2}\right)^{\mathrm{T}}$ is the vector for the amplitude and phase quadratures; we have 
assumed all the ports have identical loss equal to $\epsilon / 4$ (a quarter of the round-trip loss); $\mathbf{M}_{\text {rot }}$ is the rotation matrix of the quadratures due to free propagation in vacuum, and it is given by

$$
\mathbf{M}_{\mathrm{rot}}=e^{i \Omega \tau_{f}}\left[\begin{array}{cc}
\cos \Delta_{f} \tau_{f} & -\sin \Delta_{f} \tau_{f} \\
\sin \Delta_{f} \tau_{f} & \cos \Delta_{f} \tau_{f}
\end{array}\right]
$$

with $\tau_{f}=L_{f} / c$ being the time delay and $L_{f}$ being the filter cavity length. From these equalities, we can obtain the corresponding input-output relation between $\boldsymbol{a}$ and $\boldsymbol{b}$. Given small loss: $\eta \ll 1$, we can keep the input-output relation up to the lowest order of $\epsilon$, and get

$$
\begin{aligned}
\boldsymbol{b}= & {\left[-\sqrt{R_{f}} \mathbf{I}+T_{f}\left(\mathbf{I}-\sqrt{R_{f}} \mathbf{M}_{\mathrm{rot}}^{2}\right)^{-1} \mathbf{M}_{\mathrm{rot}}^{2}\right] \boldsymbol{a}+} \\
& \sqrt{\epsilon T_{f} / 4}\left(\mathbf{I}-\sqrt{R_{f}} \mathbf{M}_{\mathrm{rot}}^{2}\right)^{-1}\left(\mathbf{M}_{\mathrm{rot}}^{2} \boldsymbol{n}_{c}+\mathbf{M}_{\mathrm{rot}} \boldsymbol{n}_{f}+\mathbf{M}_{\mathrm{rot}} \boldsymbol{n}_{g}+\boldsymbol{n}_{d}\right) \\
& -\sqrt{\epsilon R_{f} / 4} \boldsymbol{n}_{a}+\sqrt{\epsilon / 4} \boldsymbol{n}_{b} .
\end{aligned}
$$

The term on the first line gives the cavity response in the lossless case - the quadrature rotation has a significant frequency dependence; the four terms on the second line are the losses inside the cavity, and they are amplified by the cavity around the detuning frequency $\Delta_{f}$; the two terms on the last line are the losses outside the cavity, and they are not amplified by the cavity response.

To gain an intuitive understanding, we can use the fact that the sideband frequency $\Omega$, the cavity bandwidth $\gamma_{f}$ and the detune frequency $\Delta_{f}$ are much smaller than the free spectral range, namely

$$
\Omega \tau_{f}, \gamma_{f} \tau_{f}, \Delta_{f} \tau_{f} \ll 1,
$$

with $\tau_{f} \equiv L_{f} / c$ being the propagation time. We can therefore make a Taylor expansion in terms of series of these small quantities, and obtain (in the sideband picture):

$$
\begin{aligned}
b(\Omega)= & -\frac{\Omega+\Delta_{f}-i\left(\gamma_{f}-\gamma_{\epsilon}\right)}{\Omega+\Delta_{f}+i\left(\gamma_{f}+\gamma_{\epsilon}\right)} a(\Omega) \\
& -\frac{i \sqrt{\gamma_{f} \gamma_{\epsilon}}}{\Omega+\Delta_{f}+i\left(\gamma_{f}+\gamma_{\epsilon}\right)}\left[n_{c}(\Omega)+n_{d}(\Omega)+n_{f}(\Omega)+n_{g}(\Omega)\right] \\
& -\sqrt{\epsilon / 4} n_{a}(\Omega)+\sqrt{\epsilon / 4} n_{b}(\Omega) .
\end{aligned}
$$

Here we have defined the effective bandwidth due to loss:

$$
\gamma_{\epsilon} \equiv \frac{c \epsilon}{L} .
$$

As we can see, the optical loss has two effects on the performance of the filter cavity: (i) introducing uncorrelated vacuum fluctuation that degrades the sensitivity; (ii) broadening the cavity bandwidth that prevents the use of very short cavity for filtering with desired frequency band. For a round-trip loss of order of tens of ppm, $\epsilon \sim 30 \mathrm{ppm}$, the bandwidth due to loss can be estimated as

$$
\gamma_{\epsilon} \approx 2 \pi \times 100 \mathrm{~s}^{-1}\left(\frac{L}{15 \mathrm{~m}}\right),
$$

where the loss limits the bandwidth to be larger than $100 \mathrm{~Hz}$ for a cavity length around $15 \mathrm{~m}$. For the numerical optimization to be discussed, we choose the filter cavity to be of the order of hundreds meter, and in this case, the bandwidth is mainly determined by the transmittance $T_{f}$ of the input mirror, or equivalently by $\gamma_{f}$. The loss is mainly 
important at low frequencies (coherently amplified due to cavity resonance) - it is suppressed by a factor of $\Omega^{-1}$ at high frequencies, and the total effective loss at low frequencies is given by

$$
\mathcal{E} \equiv \frac{4 \gamma_{f} \gamma_{\epsilon}}{\left(\gamma_{f}+\gamma_{\epsilon}\right)^{2}} \approx \frac{\epsilon}{T_{f}}
$$

for filter cavity bandwidth $\gamma_{f} \gg \gamma_{\epsilon}$, which recovers what has been shown in Equation C.1.

\section{Appendix D. Tolerance to parameter variations in the filter cavity}

Here we compare input filtering and output filtering in terms of tolerance to parameter uncertainties in the filter cavity. The outline of this section goes as follows: (i) we first analyze the deviation from the ideal frequency-dependent quadrature rotation due to parameter uncertainties of the filter cavity; (iii) we then show how these uncertainties influence the sensitivity for both input filtering (frequency-dependent squeezing) and output filtering (frequency dependent readout).

For a single filter cavity, the output quadratures $\left(b_{1}, b_{2}\right)$ are related to the input quadratures $\left(a_{1}, a_{2}\right)$ by [cf. Equation 12]:

$$
\left[\begin{array}{l}
b_{1} \\
b_{2}
\end{array}\right]=e^{\frac{i\left(\alpha_{+}-\alpha_{+}\right)}{2}}\left[\begin{array}{cc}
\cos \frac{\alpha_{+}+\alpha_{-}}{2} & -\sin \frac{\alpha_{+}+\alpha_{-}}{2} \\
\sin \frac{\alpha_{+}+\alpha_{-}}{2} & \cos \frac{\alpha_{+}+\alpha_{-}}{2}
\end{array}\right]\left[\begin{array}{l}
a_{1} \\
a_{2}
\end{array}\right]
$$

with $\alpha_{ \pm}$is defined as

$$
e^{i \alpha_{ \pm}} \equiv \frac{i \gamma \mp \Omega-\Delta}{i \gamma \pm \Omega-\Delta}
$$

where $\Delta$ and $\gamma$ are the detuning frequency and bandwidth of the filter cavity, respectively. If we have a chain of $N$ filter cavities, the total rotation angle $\phi_{\text {tot }}$ is given by

$$
\phi_{\text {tot }}(\Omega)=\sum_{i=1}^{N} \frac{\alpha_{+}^{(i)}(\Omega)+\alpha_{-}^{(i)}(\Omega)}{2}
$$

with $\alpha^{(i)}$ from the $i$-th filter cavity. As shown in the Appendix A of [25], by properly choosing the parameters for each cavity, one can realize any desired frequencydependent rotation angle, as long as $\tan \left(\phi_{\text {tot }}(\Omega)\right)$ is a rational function.

Suppose both the detuning frequency and bandwidth have uncertainties $\delta \Delta^{(i)}$ and $\delta \gamma^{(i)}$ :

$$
\Delta^{(i)} \rightarrow \Delta^{(i)}+\delta \Delta^{(i)}, \quad \gamma^{(i)} \rightarrow \gamma^{(i)}+\delta \gamma^{(i)} .
$$

This will induce a change $\delta \phi_{\text {tot }}$ in the total rotation angle of

$$
\begin{aligned}
\delta \phi_{\mathrm{tot}} & =\sum_{i=1}^{N} \frac{\delta \alpha_{+}^{(i)}+\delta \alpha_{-}^{i}}{2} \\
& =\sum_{i=1}^{N}-2 \gamma^{(i)} f_{+}^{(i)}(\Omega) \delta \Delta^{(i)}+2 \Delta^{(i)} f_{-}^{(i)}(\Omega) \delta \gamma^{(i)}
\end{aligned}
$$

where

$$
f_{ \pm}^{(i)}(\Omega) \equiv \frac{\gamma^{(i) 2}+\Delta^{(i) 2} \pm \Omega^{2}}{\left[\gamma^{(i) 2}+\left(\Delta^{(i)}-\Omega\right)^{2}\right]\left[\gamma^{(i) 2}+\left(\Delta^{(i)}+\Omega\right)^{2}\right]} .
$$


Quantum Limits of Interferometer Topologies for Gravitational Radiation Detection37

At low frequencies $\Omega \lesssim \gamma^{(i)}$ and for $\gamma^{(i)} \sim \Delta^{(i)}$, we have

$$
\left.\delta \phi_{\text {tot }}\right|_{\text {low freq. }}=\sum_{i=1}^{N}-\frac{\delta \Delta^{(i)}}{\Delta^{(i)}}+\frac{\delta \gamma^{(i)}}{\gamma^{(i)}}=\sum_{i=1}^{N}-\frac{\delta \Delta^{(i)}}{\Delta^{(i)}}+\frac{\delta T^{(i)}}{T^{(i)}} .
$$

Basically, the total rotation angle is just modified by the relative error in the detuning and bandwidth. In reality, such a relative error can be controlled to a level of $10^{-4}$. This means that

$$
\delta \phi_{\text {tot }} \sim 10^{-4} N .
$$

Now we look at how such an error in the quadrature rotation influences the sensitivity for input and output filtering. We focus on the tuned configuration with the input-output relation shown in Equation 6. For input filtering, the resulting spectral density is given by (cf. Equations 46-48 in [16]):

$$
S_{h}(\Omega)=\frac{h_{\mathrm{SQL}}^{2}}{2}\left(\frac{1}{\mathcal{K}}+\mathcal{K}\right)(\cosh 2 r-\cos [2(\varphi+\Phi)] \sinh 2 r) .
$$

with $\Phi=\operatorname{arccot} \mathcal{K}$. When we choose the optimal squeezing angle

$$
\varphi_{\mathrm{opt}}=-\Phi(\Omega)=-\operatorname{arccot} \mathcal{K}(\Omega),
$$

we obtain

$$
S_{h}^{\mathrm{opt}}(\Omega)=\frac{h_{\mathrm{SQL}}^{2}}{2}\left(\frac{1}{\mathcal{K}}+\mathcal{K}\right) e^{-2 r} .
$$

The uncertainties in the parameters of the filter cavity will make $\varphi$ deviate from the optimal squeezing angle $\varphi_{\text {opt }}$, i.e., $\varphi=\varphi_{\text {opt }}+\delta \varphi$ resulting in $S_{h}=S_{h}^{\text {opt }}+\delta S_{h}$. Specifically, we have

$$
\delta S_{h}(\Omega)=h_{\mathrm{SQL}}^{2}\left(\frac{1}{\mathcal{K}}+\mathcal{K}\right) \sinh (2 r) \delta \varphi^{2} .
$$

At low frequencies, we have $\left.\delta S_{h}(\Omega)\right|_{\text {low freq. }} \approx h_{\mathrm{SQL}}^{2} \sinh (2 r) \delta \phi^{2} / \mathcal{K}$.

For output filtering, the spectral density is given by [cf. Equations 56-58 in [16]] with frequency-independent phase squeezing:

$$
S_{h}(\Omega)=\frac{h_{\mathrm{SQL}}^{2}}{2 \mathcal{K}}\left[e^{-2 r}+\left(\tan \zeta-e^{2 r} \mathcal{K}\right)^{2}\right] .
$$

When we choose the optimal frequency-dependent homodyne detection angle

$$
\zeta(\Omega)=\arctan \left(e^{2 r} \mathcal{K}\right),
$$

we obtain the optimal sensitivity that is only limited by the shot noise:

$$
S_{h}^{\mathrm{opt}}(\Omega)=\frac{h_{\mathrm{SQL}}^{2}}{2 \mathcal{K}} e^{-2 r} .
$$

Similarly, variation of the readout quadrature due to uncertainties of the filter cavity parameters will induce the following change in the sensitivity:

$$
\delta S_{h}(\Omega)=\frac{h_{\mathrm{SQL}}^{2}}{\mathcal{K}}\left(1+e^{4 r} \mathcal{K}^{2}\right)^{2} \delta \zeta^{2} .
$$

Since $\mathcal{K} \gg 1$ at low frequencies, by comparing Equation D.12 and Equation D.16, we can see that $\delta S_{h}(\Omega)$ from parameter variations is much larger for the output filtering case than for input filtering. 
Quantum Limits of Interferometer Topologies for Gravitational Radiation Detection38

\section{Appendix E. Tolerance to anti-squeezing}

Here we analyze the influence of anti-squeezing (an impure squeezed state with impurity from classical noise during its preparation) on input and output filtering. In contrast to a pure squeezed state, the determinant of the noise spectral density matrix for an a state with some anti-squeezing [cf. Equation 9] is:

$$
S_{11} S_{22}-S_{12} S_{21}=\xi^{2} \geq 1,
$$

This determinant is unity for a pure squeezed state. One example of anti-squeezing is $S_{11}=\xi^{2} e^{2 r}, S_{12}=S_{21}=0$ and $S_{22}=e^{-2 r}$, which means that fluctuations in the phase quadrature are still the same as the usual pure phase squeezed state but the amplitude quadrature is larger than $e^{2 r}$ by a factor of $\xi^{2}$. In the ideal case with perfect filter cavities and no loss, such anti-squeezing will not influence the optimal sensitivity. However, when the loss and the above mentioned uncertainties in the filter cavity detuning and bandwidth are taken into account, the anti-squeezing will play an important role, and in particular, will degrade the low-frequency sensitivity.

Specifically, for the input filtering, we have

$$
\delta S_{h}(\Omega)=\frac{h_{\mathrm{SQL}}^{2}}{2}\left(\frac{1}{\mathcal{K}}+\mathcal{K}\right)\left(e^{-2 r}+e^{2 r} \xi^{2}\right) \delta \varphi^{2},
$$

At low frequencies, we can approximate this as $\delta S_{h}(\Omega) \sim h_{\mathrm{SQL}}^{2} \mathcal{K} e^{2 r} \xi^{2} \delta \varphi^{2} / 2$. For the output filtering, we have

$$
\delta S_{h}(\Omega)=\frac{h_{\mathrm{SQL}}^{2}}{\mathcal{K}}\left(1+\xi^{4} e^{4 r} \mathcal{K}^{2}\right)^{2} \delta \zeta^{2} .
$$

where we see that the output filtering is more susceptible to anti-squeezing. 